\title{
Detailed high-energy characteristics of AXP 1RXS J170849-400910
}

\section{Probing the magnetosphere using INTEGRAL, RXTE and XMM-Newton}

\author{
P.R. den Hartog ${ }^{1}$, L. Kuiper ${ }^{1}$, and W. Hermsen ${ }^{1,2}$ \\ 1 SRON, Netherlands Institute for Space Research, Sorbonnelaan 2, 3584 CA Utrecht, The Netherlands \\ 2 Sterrenkundig Instituut Anton Pannekoek, University of Amsterdam, Kruislaan 403, 1098 SJ Amsterdam, The Netherlands
}

Received 13 March 2008 / Accepted ...

\begin{abstract}
RXS J170849-400910 is one of four Anomalous X-ray Pulsars which emit persistent luminous radiation in soft $\mathrm{X}$-rays $(<10 \mathrm{keV})$ as well as in hard X-rays $(>10 \mathrm{keV})$. In this paper we present detailed spectral and temporal characteristics over the whole X-ray band. For this purpose data have been used from INTEGRAL, RXTE and XMM-Newton. The hard X-ray $(>10 \mathrm{keV})$ time-averaged total spectrum, accumulated over four years with the imager IBIS-ISGRI onboard INTEGRAL adding up to $5.2 \mathrm{Ms}$ net exposure, can be described by a power law with a photon index $\Gamma=1.13 \pm 0.06$ and extends to $\sim 175 \mathrm{keV}$. The $20-175 \mathrm{keV}$ flux is $(7.76 \pm 0.34) \times 10^{-11} \mathrm{erg} \mathrm{cm}^{-2} \mathrm{~s}^{-1}$ which exceeds the $2-10 \mathrm{keV}$ (unabsorbed) flux by a factor of $\sim 2.3$. No evidence for a spectral break is found below $300 \mathrm{keV}$. Also, no significant long-term time variability has been detected above $20 \mathrm{keV}$ on time scales of 1 and 0.5 year. Pulsed emission is measured with INTEGRAL up to $270 \mathrm{keV}$, i.e. to much higher energies than the total emission, with a detection significance of $12.3 \sigma(20-270 \mathrm{keV})$. The pulse profiles from $0.5 \mathrm{keV}$ up to $270 \mathrm{keV}$ show drastic morphology changes below $\sim 20 \mathrm{keV}$. Three different pulse components can be recognized in these pulse profiles: 1) a hard pulse peaking around phase 0.8 which contributes to the pulse profiles above $\sim 4 \mathrm{keV}, 2$ ) a softer pulse which peaks around phase 0.4 not contributing in the hard X-ray domain and 3) a very soft pulse component below $2 \mathrm{keV}$. A combined time-averaged pulsed spectrum (2.8-270 keV) from INTEGRAL, RXTE-PCA and HEXTE (collected over nine years) can be described with a soft and a hard power-law component: $\Gamma_{\mathrm{s}}=2.79 \pm 0.07$ and $\Gamma_{\mathrm{h}}=0.86 \pm 0.16$. In the pulsed spectrum extracted from a $25.5 \mathrm{ks}$ net exposure XMM-Newton observation we find a discontinuity between $2 \mathrm{keV}$ and $3 \mathrm{keV}$. Above these energies the spectrum is consistent with the spectrum taken with RXTE-PCA. The pulse profiles and the total-pulsed spectrum prove to be stable over the whole nine-years time span over which the data have been taken. Also detailed phase-resolved spectroscopy of the pulsed emission confirms the long-term stability as the spectra taken at different epochs connect smoothly. The phaseresolved spectra reveal complex spectral shapes which do not follow the shape of the total-pulsed spectrum. The spectral shape gradually changes with phase from a soft single power law to a complex multi-component shape and then to a hard single power law. The spectrum switches from a very hard $(\Gamma=0.99 \pm 0.05)$ to a very soft $(\Gamma=3.58 \pm 0.34)$ single power-law shape within a 0.1 -wide phase interval. The discontinuity measured between $2 \mathrm{keV}$ and $3 \mathrm{keV}$ with XMM-Newton is a result of a curved component. This component which is most apparent within phase interval 0.7-0.9 significantly contributes in the energy range between $4 \mathrm{keV}$ and $20 \mathrm{keV}$. It has a very steep spectrum below $5 \mathrm{keV}$ with a photon index $\Gamma \sim-1.5$. From the phase-resolved spectra we identify three independent components with different spectral shapes which together can accurately describe all phase-resolved spectra $(2.8-270 \mathrm{keV})$. The three shapes are a soft power law $(\Gamma=3.54)$, a hard power law $(\Gamma=0.99)$ and a curved shape (described with two logparabolic functions). The phase distributions of the normalizations of these spectral components form three decoupled pulse profiles. The soft component peaks around phase 0.4 while the other two components peak around phase 0.8 . The width of the curved component ( $\sim 0.25$ in phase $)$ is about half the width of the hard component. After $4 \mathrm{U}$ 0142+61, 1RXS J1708-40 is the second anomalous X-ray pulsar for which such detailed phase-resolved spectroscopy has been performed. These results give important constraints showing that three dimensional modeling covering both the geometry and different production processes is required to explain our findings.
\end{abstract}

Key words. - Stars: neutron - X-rays: individuals: 1RXS J170849-400910, 4U 0142+61 - Gamma rays: observations

\section{Introduction}

Send offprint requests to: P.R. den Hartog

Correspondence to: Hartog@sron.nl
In the past five years, several new developments have changed our view on Anomalous X-ray Pulsars (AXPs) drastically (see for a recent review Kaspi 2007). AXPs are young isolated ro- 
tating neutron stars. Their spin periods and spin derivatives indicate that the surface magnetic fields of AXPs range from $\sim 6 \times 10^{13} \mathrm{G}$ to $\sim 7 \times 10^{14} \mathrm{G}$ (assuming a dipole field; $B=$ $3.2 \times 10^{19} \sqrt{P \dot{P}} \mathrm{G}$ ). The X-ray luminosity of persistent AXPs can be orders of magnitude higher than explained by spin-down power. Nowadays, it is generally accepted that AXPs are powered by magnetic dissipation and that AXPs belong to the magnetar class (Duncan \& Thompson 1992; Thompson \& Duncan 1993, 1995, 1996; Thompson et al. 2002). To the magnetar class also belong Soft Gamma-ray Repeaters (SGRs) (Woods \& Thompson 2006; Mereghetti et al. 2007). So far, there are in total 13 confirmed magnetars known.

Traditionally, AXPs are soft X-ray sources $(\sim 0.5-10 \mathrm{keV})$ with thermal-like spectra $(k T \sim 0.4-0.7 \mathrm{keV})$ plus a soft power-law-like component with a photon index $\Gamma \sim$ $3-4$. This traditional view changed since the detections of extremely bright and persistent hard X-ray emission from AXPs by INTEGRAL ( $>20 \mathrm{keV}$ Molkov et al. 2004; Revnivtsev et al. 2004; den Hartog et al. 2004). The hard Xray spectra of these AXPs can be described with power-law models with photon indices $\Gamma \sim 0.9-1.4$ and are observed up to $230 \mathrm{keV}$ (Kuiper et al. 2004a; den Hartog et al. 2006; Kuiper et al. 2006; den Hartog et al. 2008). Because no counterparts have been found in the $\mathrm{MeV}$ gamma-ray domain by CGRO-COMPTEL $(0.75-30 \mathrm{MeV})$, these spectra are expected to show breaks. For AXP 4U 0142+61 den Hartog et al. (2008) measured the first spectral break in an AXP hard X-ray spectrum. The maximum luminosity of $4 \mathrm{U} 0142+61$ is estimated to be near $280 \mathrm{keV}$.

Moreover, Kuiper et al. (2004a, 2006) found that a significant part of the hard X-ray emission is pulsed with spectral shapes harder than the total-emission spectra. For $4 \mathrm{U} 0142+61$ pulsed emission was detected up to $160 \mathrm{keV}$ by den Hartog et al. (2008). These authors performed for $4 \mathrm{U} 0142+61$ a phase-resolved spectral analysis of the pulsed emission (i.e. without the dominating non-pulsating DC emission) in a broad band from $0.5 \mathrm{keV}$ to $160 \mathrm{keV}$ exploiting data from four space missions. In that study den Hartog et al. (2008) showed that in the pulse profiles at least three different pulse components can be recognized which appeared to have different spectra. Therefore, these pulse components are genuinely different from each other. These findings provide new constraints for modelling the underlying physical processes responsible for these spectra and the geometry of the production sites in the AXP magnetospheres. Theoretical models currently in development (e.g. Beloborodov \& Thompson 2007; Heyl \& Hernquist 2005b; Baring \& Harding 2007) do so far not explain these detailed results.

In this paper we present new high-energy results from AXP 1RXS J170849-400910 (hereafter 1RXS J1708-40). 1RXS J1708-40 has a spin period of $P=11.0 \mathrm{~s}$ and a period derivative of $\dot{P}=1.9 \times 10^{-11} \mathrm{ss}^{-1}$ (Gavriil \& Kaspi 2002). The characteristic age $(\tau=P / 2 \dot{P})$ dipolar surface magnetic-field strength of 1RXS J1708-40 inferred from these values are 9.0 kyr and $4.7 \times 10^{14} \mathrm{G}$, respectively. In the $2-10 \mathrm{keV}$ band its luminosity $L_{\mathrm{X}}$ is $1 \times 10^{35} \mathrm{erg} \mathrm{s}^{-1}$, similar to all other AXPs assuming a distance of $3.8 \mathrm{kpc}$ (Durant \& van Kerkwiik 2006a).
Soon after its discovery (Sugizaki et al. 1997) 1RXS J1708-40 has been included in the RXTE monitoring program of AXPs Gavriil \& Kaspi 2002), providing a long base-line of observations with a wealth of new information like timing parameters, pulsed-flux history and glitches (see e.g. Kaspi et al. 1999; Dib et al. 2008). For this source an intensity-hardness correlation in the soft X-ray band $(<10 \mathrm{keV})$ is claimed (Rea et al. 2007; Campana et al. 2007). An indication that this correlation also holds in the hard X-ray domain ( $>10 \mathrm{keV} \mathrm{Götz} \mathrm{et} \mathrm{al.} \mathrm{2007)}$ is not confirmed in this work.

1 RXS J1708-40 was first detected in the hard X-ray regime ( $>20 \mathrm{keV}$ ) by Revnivtsev et al. (2004) in a deep INTEGRAL Galactic centre map. Kuiper et al. (2006) discovered pulsed hard X-ray emission from this source using RXTE-PCA and HEXTE data. These authors also analysed early-mission INTEGRAL data adding up to almost $1 \mathrm{Ms}$ effective on-source exposure and found that the hard X-ray spectrum could be described by a power-law model with a photon index of $1.44 \pm$ 0.45 . Timing analysis revealed significant pulsed emission in the 20-300 keV band.

This work is a continuation of the approach followed in den Hartog et al. (2008) for AXP 4U 0142+61 applying the same methods now for 1RXS J1708-40. We address again the total high-energy window, using data collected over four years of INTEGRAL observations adding up to $\sim 5 \mathrm{Ms}$ of on-source exposure, nine years of RXTE monitoring adding up to an exposure of $\sim 600 \mathrm{ks}$ and a $\sim 25 \mathrm{ks}$ XMM-Newton observation. A significantly improved time-averaged INTEGRAL total spectrum is presented, as well as those for shorter $(\sim 1$ year and $\sim 0.5$ year) data sets to look for possible long-term time variability. Most importantly, we present the (time-averaged) total-pulsed spectra as well as (time-averaged) phase-resolved pulsed spectra from INTEGRAL, RXTE and XMM-Newton.

\section{Observations and analysis}

\subsection{INTEGRAL}

The INTErnational Gamma-Ray Astrophysics Laboratory (INTEGRAL) is a hard X-ray/soft gamma-ray (3 keV $8 \mathrm{MeV}$ ) mission which has been operational since October 2002. The payload consists of two main coded-mask imaging telescopes. 1) IBIS, Imager on Board the INTEGRAL Satellite (Ubertini et al. 2003), has a wide field of view (FOV) of $29^{\circ} \times$ $29^{\circ}$ (full-width zero response) and it has an $\sim 12^{\prime}$ angular resolution. The low-energy detector is called the INTEGRAL Soft Gamma-Ray Imager (ISGRI; Lebrun et al. 2003) and it is sensitive between $\sim 20 \mathrm{keV}$ and $\sim 300 \mathrm{keV}$. 2) The Spectrometer for INTEGRAL (SPI; Vedrenne et al. 2003) has a better spectral resolution but only a moderate imaging capability with an angular resolution of $\sim 2.5$. SPI is sensitive between $\sim 20 \mathrm{keV}$ and $\sim 8 \mathrm{MeV}$ and has a FOV of $35^{\circ} \times 35^{\circ}$ (full-width zero response).

For the best sky map reconstruction INTEGRAL performs observations in dither patterns (Jensen et al. 2003) besides the occasional staring observations. Typical pointings (Science Windows, ScWs) last $\sim 1800-3600 \mathrm{~s}$. Due to the limited visibility windows on the Galactic-centre region, the observations 
Table 1. Summary of the INTEGRAL observations of 1RXS J1708-40. The following information is given: the revolution intervals, the observations time spans both in MJD as in calendar dates, the number of ScWs, exposure times $\left(t_{\text {exp }}\right)$, and the effective on-source exposure $\left(t_{e f f}\right)$.

\begin{tabular}{llllrrrr}
\hline \hline Rev. & \multicolumn{3}{c}{ Time span } & & ScWs & $t_{\text {exp }}(\mathrm{ks})$ & $t_{\text {eff }}(\mathrm{ks})$ \\
\hline $037-485$ & $52668-54013$ & Jan. 29, & 2003- Oct. 5, & 2006 & 5161 & 11959.1 & 5191.0 \\
\hline $037-063$ & $52668-52751$ & Jan. 29, & $2003-$ Apr. 22, & 2003 & 706 & 1394.3 & 700.7 \\
$100-120$ & $52859-52920$ & Aug. 8, & 2003- Oct. 8, & 2003 & 717 & 1843.6 & 709.0 \\
$164-185$ & $53051-53115$ & Feb. 16, & 2004- Apr. 20, & 2004 & 592 & 1317.0 & 519.0 \\
$222-246$ & $53225-53298$ & Aug. 8, & 2004- Oct. 20, & 2004 & 698 & 1590.3 & 618.7 \\
$283-307$ & $53407-53481$ & Feb. 6, 6, 2005- Apr. 21, & 2005 & 1027 & 2256.9 & 1116.4 \\
$345-366$ & $53594-53655$ & Aug. 12, & $2005-$ Oct. 12, & 2005 & 470 & 1009.6 & 450.5 \\
$399-431$ & $53756-53850$ & Jan. 21, & 2006- Apr. 25, & 2006 & 517 & 1405.8 & 534.0 \\
$466-485$ & $53960-54013$ & Aug. 13, & 2006- Oct. 5, & 2006 & 434 & 1141.6 & 542.6 \\
\hline
\end{tabular}

form sets of consecutive orbital revolutions (Revs). In Table 1 a list of these sets are given with the total exposure time for the selected ScWs for which 1RXS J1708-40 was within an angle of 14.5 from the pointing direction of IBIS, as well as the effective on-source exposure, which is reduced due to off-axis viewing angles.

The region where 1RXS J1708-40 is located is often observed with INTEGRAL as it is $\sim 15^{\circ}$ from the Galactic centre. After four years of observations the total exposure on this region adds up to $12 \mathrm{Ms}$ taken in 5161 pointings, after screening for Solar flares and erratic count-rates due to the passages of the spacecraft through the Earth's radiation belts. Taking into account the off-axis angles the effective on-source exposure is 5.2 Ms.

\subsubsection{INTEGRAL spectra from spatial analysis}

The spectral analysis of the IBIS-ISGRI data follows the same procedures as applied in den Hartog et al. (2008). The shadowgrams of $5161 \mathrm{ScWs}$ are deconvolved into sky images in 20 energy intervals using the Off-line Scientific Analysis software package version 5.1 (OSA; see Goldwurm et al. 2003). The energy intervals are exponentially binned between $20 \mathrm{keV}$ and $300 \mathrm{keV}$. At the source position of 1RXS J1708-40 averaged count rates are determined for all energy bins. Photon fluxes are generated by normalizing the AXP count-rates to the Crab count-rates determined from INTEGRAL Crab observations during Rev. 102, and the Crab spectrum. The spectral shape of the Crab spectrum we adopted is a curved power-law shape

$F_{\gamma}=1.5703(14) \times\left(E_{\gamma} / 0.06335\right)^{-2.097(2)-0.0082(16) \times \ln \left(E_{\gamma} / 0.06335\right)}(1)$

(Eq. 2 in Kuiper et al. 2006), where $F_{\gamma}$ is expressed in $\mathrm{ph} /\left(\mathrm{cm}^{2} \mathrm{~s} \mathrm{MeV}\right)$ and $E_{\gamma}$ in MeV. For fitting these (and other) spectra the software package $X$-spec version 12.3 Arnaud 1996) and private software are used. All errors quoted in this paper are $1 \sigma$ errors.

For the INTEGRAL observations up to Rev. 366 SPI spectral analysis is performed above $140 \mathrm{keV}$, from where the sensitivity of SPI is comparable or higher than ISGRI. In the $140 \mathrm{keV}-1 \mathrm{MeV}$ energy range the data were binned in 4 energy bins. From the total-exposure sky maps source fluxes have
Table 3. Summary of the RXTE observations of 1RXS J170840. The following information is given: the observation IDs, time spans of the observations in calendar dates, PCA exposure times for PCU-2 in units ks.

\begin{tabular}{|c|c|c|c|}
\hline Obs. ID. & Time span & & $t_{\mathrm{PCA}}$ \\
\hline ALL & Jan. 12,1998 - Nov. 16, & 2006 & 606.512 \\
\hline Set A & Jan. 12,1998 - Oct. 26, & 2003 & 310.480 \\
\hline Set B & Nov. 1, 2003 - Nov. 16, & 2006 & 296.032 \\
\hline \multicolumn{4}{|c|}{ Set A } \\
\hline 30125 & Jan. 12, 1998 - Jan. 08, & 1999 & 59.896 \\
\hline 40083 & Feb. 6, $1999-$ Mar. 11, & 2000 & 52.568 \\
\hline 50082 & Apr. 21, $2000-$ May 12, & 2001 & 34.576 \\
\hline 60069 & May 6, $2001-$ Feb. 20, & 2002 & 24.544 \\
\hline 60412 & May 20, 2001 - May. 23, & 2001 & 9.928 \\
\hline 70094 & Apr. 2, $2002-$ Mar. 20, & 2003 & 55.600 \\
\hline 80098 & $\begin{array}{cc}\text { Apr. 16, } 2003 \text { - Oct. 26, } & \text { Set B }\end{array}$ & 2003 & 73.368 \\
\hline 80098 & Nov. 1, 2003 - Feb. 23, & 2004 & 26.416 \\
\hline 90076 & Feb. 28, 2004 -Feb. 28, & 2005 & 126.784 \\
\hline 91070 & Mar. 6, $2005-$ Feb. 25, & 2006 & 79.000 \\
\hline 92006 & Mar. 5, 2006 - Nov. 16, & 2006 & 63.832 \\
\hline
\end{tabular}

been extracted for every energy bin using a maximum likelihood fitting procedure that considers the instrumental response of SPI (Knödlseder 2004). Also here the measured flux is normalized to the Crab spectrum, which has been obtained using SPI observations of Revs. 43-45.

\subsubsection{INTEGRAL timing analyses}

For the IBIS-ISGRI timing analysis of AXP 1RXS J1708-40 we followed earlier applied procedures (Kuiper et al. 2006; den Hartog et al. 2008). All available data taken between Rev. 037 and 485 were used (see Table 11). Photons were selected only from non-noisy detector elements that could be illuminated by the source through the open mask elements by more than $25 \%$. After correcting for instrumental, onboard processing and ground-station time delays (Walter et al. 2003), the event arrival times are Solar-system barycentered (JPL DE200 Solar-system ephemeris) adopting the best known source position (Israel et al. 2003). The barycentered events are folded 
Table 2. Phase-coherent ephemerides - derived from RXTE-PCA monitoring data - valid for the analysed INTEGRAL observations.

\begin{tabular}{|c|c|c|c|c|c|c|c|c|}
\hline AXP & $\begin{array}{l}\text { Start } \\
{[\mathrm{MJD}]}\end{array}$ & $\begin{array}{l}\text { End } \\
{[\mathrm{MJD}]}\end{array}$ & $\begin{array}{l}\text { INTEGRAL } \\
\text { range (Revs) }\end{array}$ & $\begin{array}{l}t_{\text {Epoch }} \\
{[\mathrm{MJD}, \mathrm{TDB}]}\end{array}$ & $\begin{array}{l}v \\
{[\mathrm{~Hz}]}\end{array}$ & $\begin{array}{l}\dot{v} \\
\times 10^{-13}\left[\mathrm{~Hz} \mathrm{~s}^{-1}\right]\end{array}$ & $\begin{array}{l}\ddot{v} \\
\times 10^{-22}\left[\mathrm{~Hz} \mathrm{~s}^{-2}\right]\end{array}$ & $\Phi_{0}$ \\
\hline 1RXS J1708-40 & 52590 & 52960 & $009-133$ & 52590 & 0.0908982198657 & -1.58268 & 0.00 & 0.4333 \\
\hline 1RXS J1708-40 & 53050 & 53325 & $163-255$ & 53050 & 0.0908919724436 & -1.62994 & 2.75 & 0.5025 \\
\hline 1RXS J1708-40 & 53321 & 53520 & $254-320$ & 53377 & 0.0908875116829 & -1.49563 & -34.1 & 0.7559 \\
\hline 1RXS J1708-40 & 53555 & 53690 & $332-377$ & 53555 & 0.0908852012859 & -1.77561 & 36.4 & 0.8587 \\
\hline 1RXS J1708-40 & 53741 & 54055 & $395-499$ & 53741 & 0.0908825979045 & -1.57228 & -0.813 & 0.1842 \\
\hline
\end{tabular}

using a phase-connected ephemeris (see Table 2 and Sect. 2.2 for the ephemeris creation). The (TDB) time to pulse phase conversion taking into account consistent phase alignment for each ephemeris is provided by the following formula:

$\Phi(t)=v \cdot\left(t-t_{\text {Epoch }}\right)+\frac{1}{2} \dot{v} \cdot\left(t-t_{\text {Epoch }}\right)^{2}+\frac{1}{6} \ddot{v} \cdot\left(t-t_{\text {Epoch }}\right)^{3}-\Phi_{0} \cdot$

For the pulse profiles, detection significances are estimated by applying a $\mathrm{Z}_{n}^{2}$ test on bin-free pulse-phase distributions. (Buccheri et al. 1983). Truncated Fourier series with two harmonics are used to fit the pulse profiles, where the minimum of these fits are defined as the DC level.

The total-pulsed spectrum is derived by obtaining the number of excess counts above the DC level from each pulse profile for all considered energy bands. These excess counts are normalized in Crab units (by applying the same procedure for the Crab pulsar) and are converted into photon fluxes using a Crabpulsar spectrum model (Eq. 3 in Kuiper et al. 2006);

$F_{\gamma}=0.4693(21) \times\left(E_{\gamma} / 0.04844\right)^{-1.955(7)-0.0710(78) \times \ln \left(E_{\gamma} / 0.04844\right)}$

The analysis to create phase-resolved spectra (Sect. 3.3.2) is similar. However, for the phase-resolved spectra only the excess counts within selected phase intervals are used.

\subsection{RXTE}

For the timing analysis of the INTEGRAL data the regular monitoring observations with the Rossi X-ray Timing Explorer (RXTE) during the INTEGRAL observations are of great importance (Gavriil \& Kaspi 2002), because the INTEGRAL source count rate is too low to independently detect the pulsation. This is possible with the data from the Proportional Counter Array (PCA; Jahoda et al. 1996) aboard RXTE. The PCA is a non-imaging instrument sensitive in the $2-60 \mathrm{keV}$ energy range and it consists of five collimated units (PCUs 0-4) with a $\sim 1^{\circ}$ FOV. Using these RXTE-PCA monitoring data we created accurate phase-connected ephemerides valid during the INTEGRAL observations. The details of the ephemerides can be found in Table 2. The pulsar phase was set arbitrarily to zero where the pulse profile for energies between $\sim 2.5$ and $10 \mathrm{keV}$ reached a sharp minimum (see e.g. Fig. 7)B). This is a different choice than made in Kuiper et al. (2006), resulting in a phase shift in the profiles of $\sim 0.2$.

For the study of the pulsed emission of 1RXS J1708-40 with RXTE-PCA, we have used all publicly available data spanning nine years of observations. The data are analysed in two sets. Set A contains the data which have been used by Kuiper et al. (2006) and were taken over a period of almost six years. Most of these data were taken before the start of the INTEGRAL observations. Set B covers the remaining part of the publicly available data taken during INTEGRAL operations and span about three years. All data used in this work have been listed in Table 3. It can be seen that Set A and B have similar total exposures on 1RXS J1708-40.

The procedures for the RXTE spectral-timing analysis as outlined by Kuiper et al. (2006), and also applied in den Hartog et al. (2008), have been followed. The pulse profiles for any selected energy band can be described sufficiently accurate by truncated Fourier series with three harmonics above constant DC levels. The excess (pulsed) counts above these DC levels can then be converted into flux units using PCU exposure-weighted response matrices (see e.g. Sect. 3.2 of Kuiper et al. 2006). To derive unabsorbed PCA time-averaged total-pulsed and phase-resolved spectra, a Galactic absorption column $N_{\mathrm{H}}=1.47 \times 10^{22} \mathrm{~cm}^{-2}$ was used (fixed value), derived from XMM-Newton spectral fits (see Sect. 3.1.3).

The High-Energy X-Ray Timing Experiment (HEXTE; 15$250 \mathrm{keV}$; Rothschild et al. 1998) aboard RXTE has also been used to extend the PCA spectral-timing study to higher energies. HEXTE consists of two independent detector clusters ( 0 and 1 ) each with a $\sim 1^{\circ}$ FOV. HEXTE allows for detailed consistency checks with the INTEGRAL results obtained for similar energy windows. Due to the co-alignment of HEXTE and the PCA, the same long monitoring observations of 1RXS J1708-40 can be used for both instruments. Fully consistent procedures have been applied for the spectral-timing analysis, including the use of the ephemerides listed in Table 2 , and following the same procedures for deriving pulsed counts and fluxes of the pulsed emission. On 1RXS J1708-40 the total deadtime corrected exposures for clusters 0 and 1 are 239.04 ks and $259.50 \mathrm{ks}$, respectively.

\subsection{XMM-Newton}

XMM-Newton (Jansen et al. 2001) has been operational since early 2000. Onboard are three CCD cameras for X-ray imaging, namely two EPIC (European Photon Imaging Camera) MOS (Turner et al. 2001) cameras and one EPIC-PN (Strüder et al. 2001) camera. All cameras have a FOV of $\sim 30^{\prime}$ and are sensitive in the energy range $\sim 0.3-12 \mathrm{keV}$.

We have (re)analysed a publicly available $45 \mathrm{ks}$ XMMNewton observation on 1 RXS J1708-40. The data were taken in 
Table 4. Summary of the power-law model fits to the INTEGRAL-IBIS-ISGRI total-spectra of 1RXS J1708-40 summing observations taken in intervals of $\sim 1$ year and for the total exposure. For each spectrum are given the revolution interval over which 1RXS J1708-40 observations are summed, the photon index, the $20-150 \mathrm{keV}$ flux and $\chi_{r}^{2}$.

\begin{tabular}{lccr}
\hline \hline Rev. & $\Gamma$ & $\begin{array}{c}F_{20-150 \times 10^{-11}} \\
{\left[\mathrm{erg} \mathrm{cm}^{-2} \mathrm{~s}^{-1}\right]}\end{array}$ & $\chi_{r}^{2}$ (dof) \\
\hline $037-120$ & $1.18 \pm 0.14$ & $5.47 \pm 0.43$ & $1.64(14)$ \\
$164-246$ & $1.40 \pm 0.15$ & $5.97 \pm 0.55$ & $1.09(14)$ \\
$283-366$ & $0.97 \pm 0.12$ & $7.13 \pm 0.44$ & $0.52(14)$ \\
$399-485$ & $1.00 \pm 0.21$ & $8.72 \pm 0.61$ & $1.67(14)$ \\
\hline $037-485$ & $1.13 \pm 0.06$ & $6.61 \pm 0.23^{\dagger}$ & $1.13(14)$ \\
${ }^{\dagger} F_{20-175}=(7.76 \pm 0.34) \times 10^{-11} \mathrm{erg} \mathrm{cm}^{-2} \mathrm{~s}^{-1}$ \\
\hline
\end{tabular}

the night of August 28 to 29, 2003 (Obs. Id 0148690101). The data are analysed using SAS v. 7.0 and the latest calibration files that were available (May 2007). Next, we considered the EPIC-PN which operated in small-window mode with medium filter. In this mode the maximum time resolution is $6 \mathrm{~ms}$. These data are checked for solar (soft proton) flares by creating a light curve with events with energies larger than $10 \mathrm{keV}$. A countrate distribution was created from this light curve to which a Gaussian was fitted to determine the mean count rate of the high-energy photons. Good-Time Intervals (GTIs) were created allowing only time stamps for which the count rate was lower than 0.078 counts per second which corresponds to the fitted mean count rate plus three times the width of the distribution $(3 \sigma)$. The GTIs add up to $25.5 \mathrm{ks}$ of exposure. All single and double events, i.e. patterns less than and equal four, are selected within the energy range $0.3 \mathrm{keV}$ to $12 \mathrm{keV}$. The arrival times of the selected events are barycentered.

There was no need to correct for pile-up effects as the count rate of 1RXS J1708-40 was low enough for the PN in small window mode with medium filter. To extract the source counts a circular extraction region with a radius of $35^{\prime \prime}$ was used. As this source is not so bright it was possible to select a background extraction region from the small window with a radius of 45". No out-of-time event correction has been applied as the effect is approximately $1 \%$. The extracted spectrum has been binned oversampling the energy resolution by a factor of three and then rebinned occasionally (for energies $\gtrsim 5 \mathrm{keV}$ ) to ensure a minimum of 25 counts per bin.

For timing analysis, the barycentered events are folded using an appropriate ephemeris (ephemeris 1 in Table 2). To create pulsed spectra pulsed excess counts are extracted as described in Sect. 2.1.2, fitting the pulse profiles with truncated Fourier series with the first three harmonics above a constant background.

\section{Results}

In this section we present results from INTEGRAL, XMMNewton and RXTE. First, we present the total (pulsed plus DC) spectrum using INTEGRAL data above $20 \mathrm{keV}$ and look for possible long-term time variability. Then we derive the com-

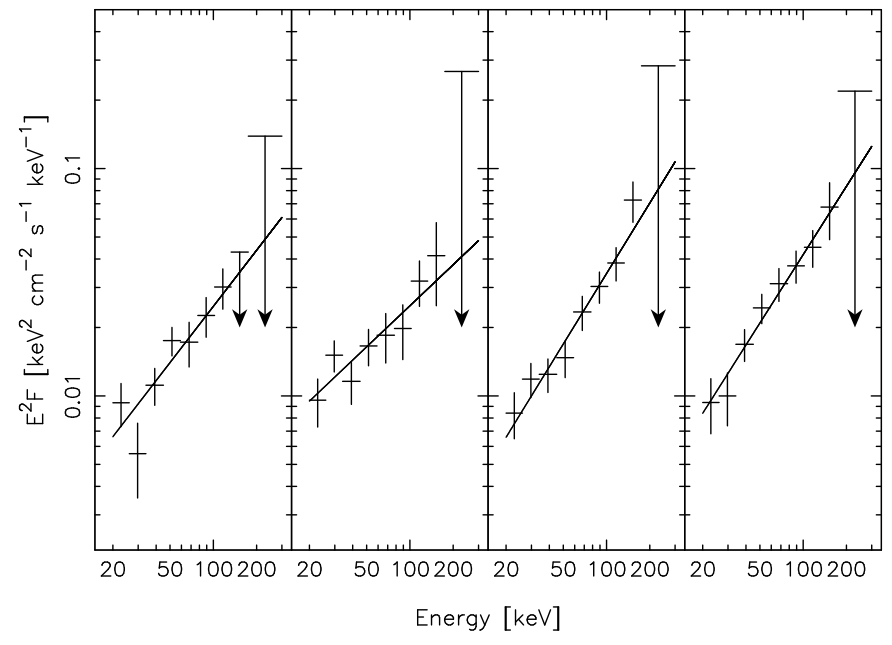

Fig. 1. Total-flux (pulsed + DC) spectra of 1RXS J1708-40 collected during four one-year intervals of INTEGRAL observations, corresponding to revolution intervals (from left to right); 037-120, 164-246, 283-366 and 399-485. The upper limits are $2 \sigma$. The model lines are single power-law fits (see Table 4 ).

plementary XMM-Newton total spectrum below $12 \mathrm{keV}$. RXTE is a non-imaging mission and due to the presence of nearby strong sources and large gradients in the Galactic ridge emission (Valinia \& Marshall 1998), it can in this work only be used for studying the timing signal of 1RXS J1708-40. The pulse profiles are then presented and compared for all three missions. Finally, the results from spectral timing analyses in the form of total-pulsed spectra and phase-resolved spectra for all data sets are presented and discussed in detail. This leads to the identification of genuinely different components contributing to the pulsed high-energy emission.

\subsection{Total spectra and long-term variability}

\subsubsection{INTEGRAL ISGRI persistent time-averaged spectra and long-term variability}

Listed in Table 1 are eight sets of INTEGRAL observations, each covering about half a year. 1RXS J1708-40 is not bright in hard X-rays, therefore we first performed the spectral analysis averaging observations over one-year time intervals to obtain significant flux values till above $100 \mathrm{keV}$. The resulting four spectra are shown in Fig. 11. They can be described satisfactorily with single power-law models. The fit results are listed in Table 4. The power-law indices range from $0.97 \pm 0.12$ to $1.40 \pm$ 0.15 and the $20-150 \mathrm{keV}$ fluxes range from $(5.47 \pm 0.43) \times 10^{-11}$ to $(8.72 \pm 0.61) \times 10^{-11} \mathrm{erg} \mathrm{cm}^{-2} \mathrm{~s}^{-1}$ which can be seen as a indication for time variability. When we compare these yearly averages with the long-term four year average (see Sect. 3.1.2), we find that the spectra taken in Revs. 037-120 and 399-485 are most deviating from this time-averaged spectrum, i.e. at the $\sim 2 \sigma$ and $\sim 3 \sigma$ level, respectively (see error contours in Fig. 2).

Alternatively, the standard deviations $(s=$ $\sqrt{\left.1 /(n-1) \sum_{i}\left(x_{i}-\bar{x}\right)^{2}\right)}$ for the power-law indices and the 20-150 keV fluxes relative to the weighted mean are 0.20 and $1.46 \times 10^{-11} \mathrm{erg} \mathrm{cm}^{-2} \mathrm{~s}^{-1}$, respectively. Therefore, the power- 


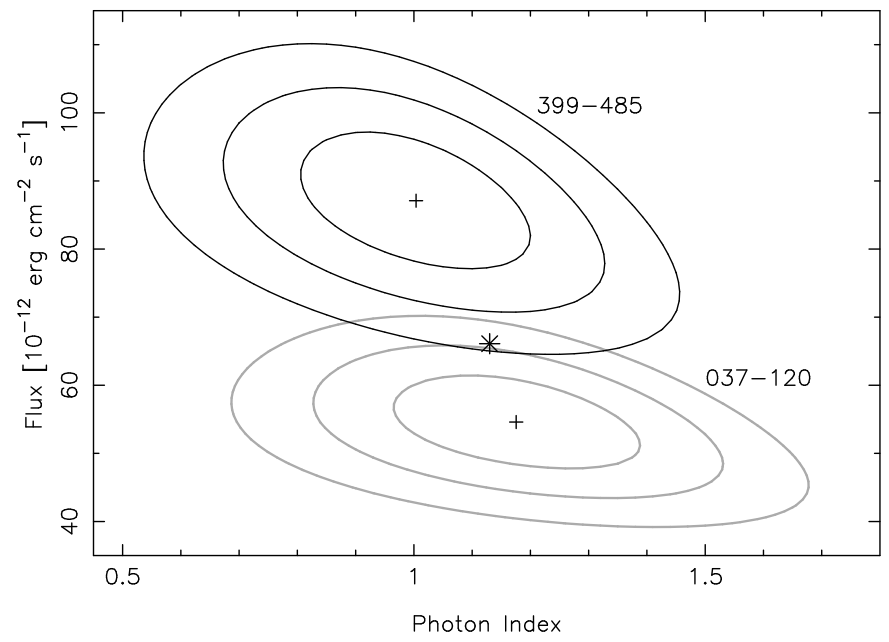

Fig. 2. Error contours $(1 \sigma, 2 \sigma$ and $3 \sigma)$ of the power-law model fits to the total spectrum $(20-300 \mathrm{keV})$ for observations 037 120 and 399-485 compared to the total time-averaged fit (037485). Out of the four observations (each averaged over one year) these two differ most from the four-year time-averaged spectrum.

law shape is stable within $18 \%(1 \sigma)$ and the $20-150 \mathrm{keV}$ flux is stable within $22 \%(1 \sigma)$.

To explore the indication for variability further, we also fitted the spectra of the half-year long time sets (Table 1) plus a more recent short, lower-statistics observation (Revs 534-542, Feb. 27 - Mar. 25, 2007, 173 ScWs, 307 ks effective on-source exposure) with single power-law models, but over the narrower energy band 20-70 keV. The results are plotted in Fig. 3. The 20-70 keV flux measurement for Revs. 399-431 is the only one that deviates at a $3 \sigma$ level from the time-average value. Since this is one single $3 \sigma$ deviation for nine measurements, we consider this only as an indication for time variability.

The bottom panel of Fig. 3 shows no indication for significant variation in spectral index, either. Israel et al. (2007) and Dib et al. (2008) reported the detection of glitches and candidate glitches of 1RXS J1708-40 during the INTEGRAL operations, the epochs are indicated in Fig. 3. This figure shows that there is no indication for an obvious change in spectral slope nor flux triggered by one of the glitches. Possibly there is a change in spectral slope after the glitch on MJD 53366. However, the difference between $\Gamma=1.27 \pm 0.10$ and $\Gamma=0.98 \pm 0.08$, the average values before and after the glitch, is not significant $(2.3 \sigma)$.

Götz et al. (2007) analysed part of the INTEGRAL data used in this work, they did not use Revs. 164-185, 345-366 and 399-431, and claim the detection of long-term hard Xray $(20-70 \mathrm{keV})$ variability based on an indication for lower flux values in the INTEGRAL measurements after the second candidate glitch (epoch marked in Fig. 3), correlated with lower fluxes measured with Swift in the $1-10 \mathrm{keV}$ band (their Fig. 1). However, our more complete analysis including full spectral fitting of the data in stead of using merely count rates does not confirm the reported trend in the INTEGRAL data for 1RXS J1708-40 (see Fig. 3). Therefore, we do not support the

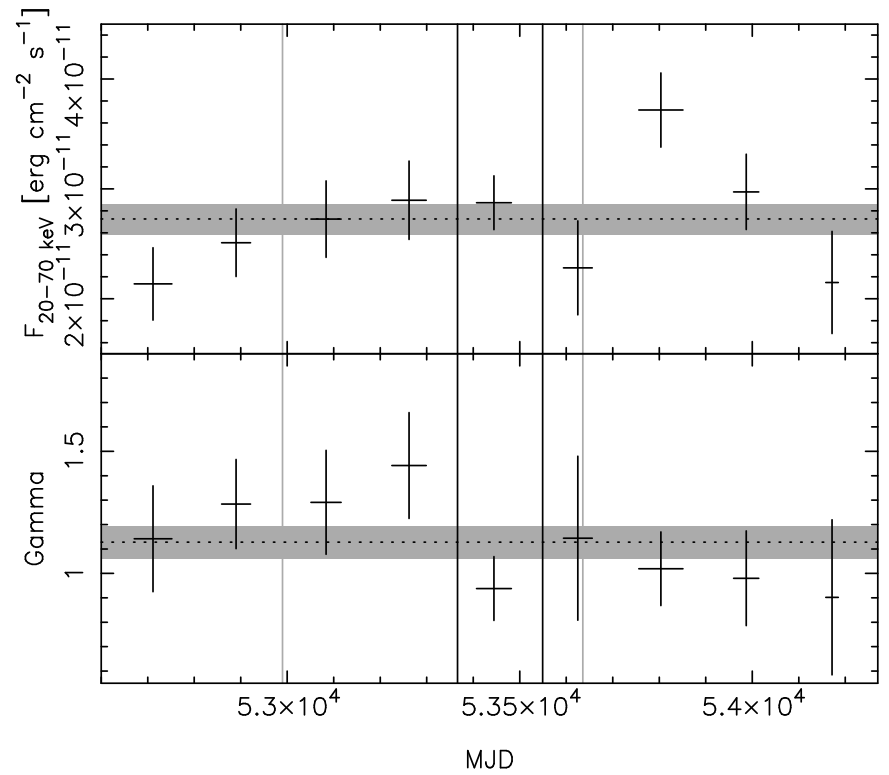

Fig. 3. Fluxes (20-70 keV) and photon indices for the half-year time sets specified in Table 1 and one shorter observation (see Sect. 3.1.1. Indicated as horizontal dotted lines are the timeaveraged values with their $1 \sigma$ errors (grey bands). Glitches and candidate glitches (Israel et al. 2007; Dib et al. 2008) are indicated as vertical black and grey lines, respectively.

claimed correlated variability between the hard X-ray and soft X-ray emission from 1RXS J1708-40.

\subsubsection{INTEGRAL time-averaged total spectrum}

Combining all the data listed in Table 1 a time-averaged spectrum is extracted using 5.2 Ms on-source exposure time (Fig. 4). 1RXS J1708-40 has been detected with high significance up to $175 \mathrm{keV}$ with a $4.2 \sigma$ detection significance in the $152-175 \mathrm{keV}$ band. Fitting this spectrum with a single power law yields an excellent fit $\left(\chi_{r}^{2}=1.13\right.$ with 14 dof $)$ with a photon index $\Gamma=1.13 \pm 0.06$ and a $20-150 \mathrm{keV}$ flux of $(6.61 \pm 0.23) \times 10^{-11} \mathrm{erg} \mathrm{cm}^{-2} \mathrm{~s}^{-1}$. Its $20-175 \mathrm{keV}$ luminosity is $L_{20-175}=1.34 \times 10^{35} \mathrm{erg} \mathrm{s}^{-1}$ assuming a distance of $3.8 \mathrm{kpc}$ (Durant \& van Kerkwijk 2006a). It is remarkable that there is no hint for a spectral break up to the highest energies in our INTEGRAL-ISGRI spectrum, particularly when compared to the reported COMPTEL upper limits for energies above 750 $\mathrm{keV}$ (Kuiper et al. 2006), which impose a spectral break between $100 \mathrm{keV}$ and $750 \mathrm{keV}$ (see Fig. 4).

Unfortunately, it turned out that 1RXS J1708-40 is not bright enough above $\sim 100 \mathrm{keV}$ to be detected with INTEGRAL-SPI, even for this long exposure. In Fig. 4 three $2 \sigma$ upper limits derived from SPI spatial analysis are added. These limits are all above the extrapolation of the power-law fit to the ISGRI flux values, contrary to the case of $4 \mathrm{U} 0142+61$, for which SPI upper limits provided evidence for the presence of a spectral break (den Hartog et al. 2008). 


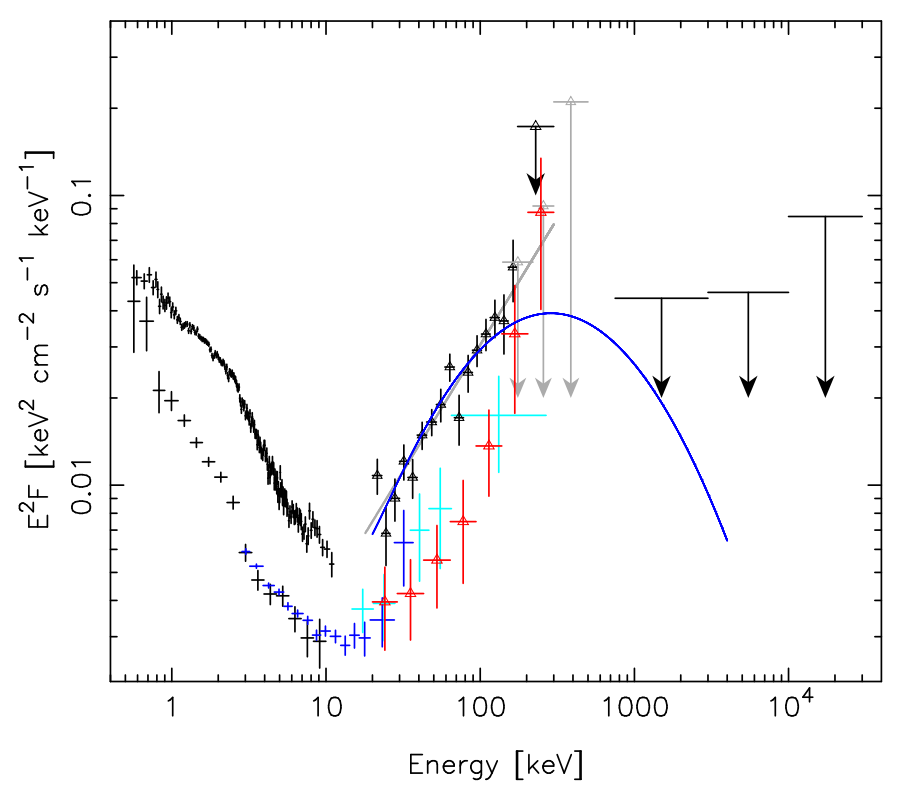

Fig. 4. High-energy spectra of 1RXS J1708-40. In this figure the following is plotted: the unabsorbed total spectra of XMMNewton $(<12 \mathrm{keV})$ and INTEGRAL (with triangle markers) in black, also in black three COMPTEL upper limits (Kuiper et al. 2006), three INTEGRAL-SPI upper limits in grey (with triangle markers); also in grey a power-law fit to the INTEGRALIBIS spectrum, in blue a logparabolic fit to the INTEGRALIBIS, SPI and COMPTEL data, total pulsed spectra of XMMNewton in black, RXTE-PCA and HEXTE are shown in blue and aqua, and the total pulsed spectrum of INTEGRAL-ISGRI in red (with triangle markers).

Following den Hartog et al. (2008) we have fitted all INTEGRAL and COMPTEL spectral information (including limits) with a logparabolic function;

$F=F_{0} \times\left(\frac{E}{E_{0}}\right)^{-\alpha-\beta \cdot \ln \left(\frac{E}{E_{0}}\right)}$

where $E_{0}$ (in units $\mathrm{keV}$ ) is the pivot energy to minimize correlations between the parameters and $F_{0}$ is the flux (in units ph $\mathrm{cm}^{-2} \mathrm{~s}^{-1} \mathrm{keV}^{-1}$ ) at $E_{0}$. This function is a power-law if the curvature parameter $\beta$ is equal to zero. Assuming this spectral shape we get an acceptable broad-band $(20 \mathrm{keV}-30 \mathrm{MeV})$ fit with best-fit parameters $\alpha=1.637 \pm 0.049, \beta=0.261 \pm 0.035$ and $F_{0}=(1.68 \pm 0.08) \times 10^{-6} \mathrm{ph} \mathrm{cm}^{-2} \mathrm{~s}^{-1} \mathrm{keV}^{-1}$ at $E_{0}=143.276$ $\mathrm{keV}$. The peak energy $E_{\text {peak }}$ is $287_{-45}^{+75} \mathrm{keV}$. This value lies remarkably close to the peak energy found for $4 \mathrm{U} 0142+61$ (i.e. $279_{-41}^{+65} \mathrm{keV}$; den Hartog et al. 2008). In Fig. 4 both the powerlaw and the logparabolic fit are drawn.

\subsubsection{XMM-Newton total spectrum}

For energies below $12 \mathrm{keV}$ we extracted the absorbed total (pulsed + DC) spectrum using XMM-Newton EPIC-PN data (see Sect. 2.3). In order to obtain an estimate for the Galactic absorption column $\left(N_{\mathrm{H}}\right)$ we fitted the spectrum globally with a canonical logparabolic function, including fixed INTEGRAL parameters for the hard X-ray contribution above

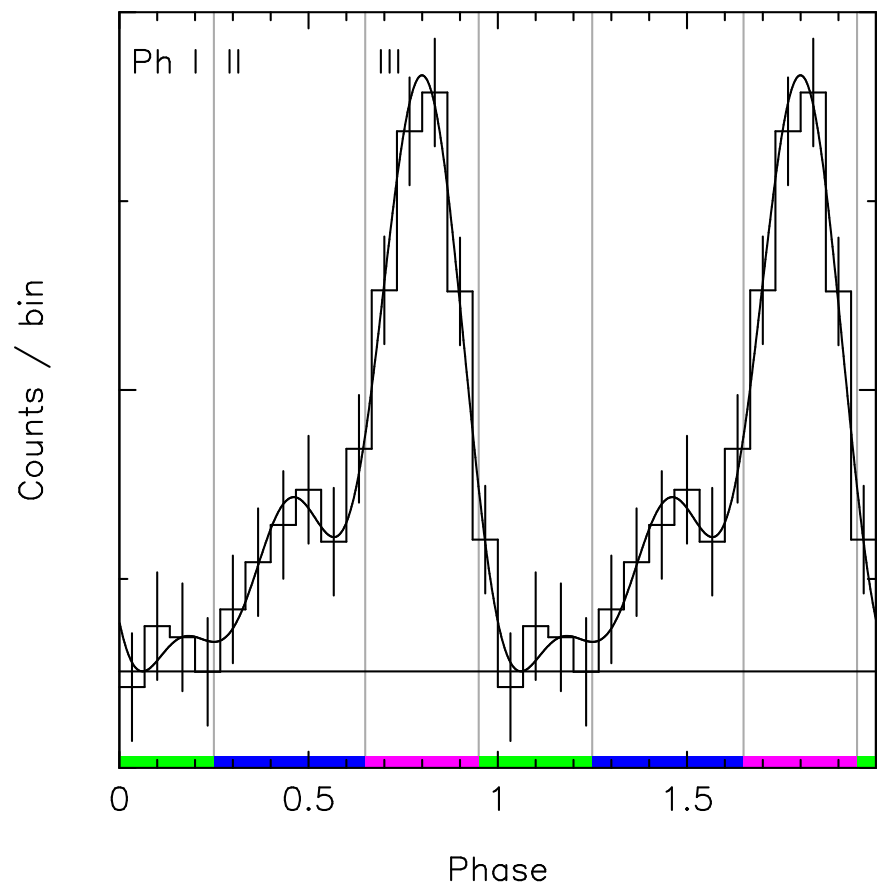

Fig. 5. IBIS-ISGRI pulse profile of 1RXS J1708-40 between 20 $\mathrm{keV}$ and $270 \mathrm{keV}$. This profile has a $12.3 \sigma$ significance using a $\mathrm{Z}_{3}^{2}$ test (fit shown as a solid curve). The fitted DC level is indicated with an horizontal line. The grey lines and the colours indicate three phase intervals Ph I, II and III (see Table 6). The colours are consistently used in this paper in figures showing results of phase-resolved analyses (see Sect. 3.3.2).

$\sim 8 \mathrm{keV}$. We derive an $N_{\mathrm{H}}$ of $(1.47 \pm 0.02) \times 10^{22} \mathrm{~cm}^{-2}$, which can be compared with the value $(1.36 \pm 0.03) \times 10^{22}$ $\mathrm{cm}^{-2}$ obtained by Rea et al. (2005) fitting the same XMMNewton data with an absorbed black-body plus a powerlaw model. Durant \& van Kerkwijk (2006b) used a modelindependent approach analysing X-ray grating spectra taken with the Reflection Grating Spectrometer (den Herder et al. 2001) onboard XMM-Newton. Their value for $N_{\mathrm{H}}$ of $(1.40 \pm$ $0.4) \times 10^{22} \mathrm{~cm}^{-2}$ is consistent with both estimates. We adopted $N_{\mathrm{H}}=1.47 \times 10^{22} \mathrm{~cm}^{-2}$ in this work for the XMM-Newton and RXTE analyses. The total unabsorbed spectrum is shown in Fig. 4. The $2-10 \mathrm{keV}$ unabsorbed flux is $(3.398 \pm 0.012) \times 10^{-11}$ erg $\mathrm{cm}^{-2} \mathrm{~s}^{-1}$. The error is statistical only. The $2-10 \mathrm{keV}$ unabsorbed fluxes for $N_{\mathrm{H}}=1.40 \times 10^{22}$ and $1.36 \times 10^{22} \mathrm{~cm}^{-2}$ are $(3.361 \pm 0.009) \times 10^{-11}$ and $(3.339 \pm 0.013) \times 10^{-11} \mathrm{erg} \mathrm{cm}^{-2} \mathrm{~s}^{-1}$, respectively. These values are within $2 \%$ of our value.

\subsection{Pulse profiles}

\subsubsection{INTEGRAL and XMM-Newton pulse profiles}

Kuiper et al. (2006) showed for the first time pulsed hard Xray emission ( $>10 \mathrm{keV}$ ) from 1RXS J1708-40 using data from RXTE-PCA, RXTE-HEXTE and INTEGRAL-ISGRI. For the INTEGRAL pulse profiles $\sim 1.4 \mathrm{Ms}$ on-source exposure was used, resulting in a $5.9 \sigma$ detection for energies $20-300 \mathrm{keV}$. In this work, we present INTEGRAL pulse profiles using 75.2 Ms on-source exposure. The result is a very much im- 


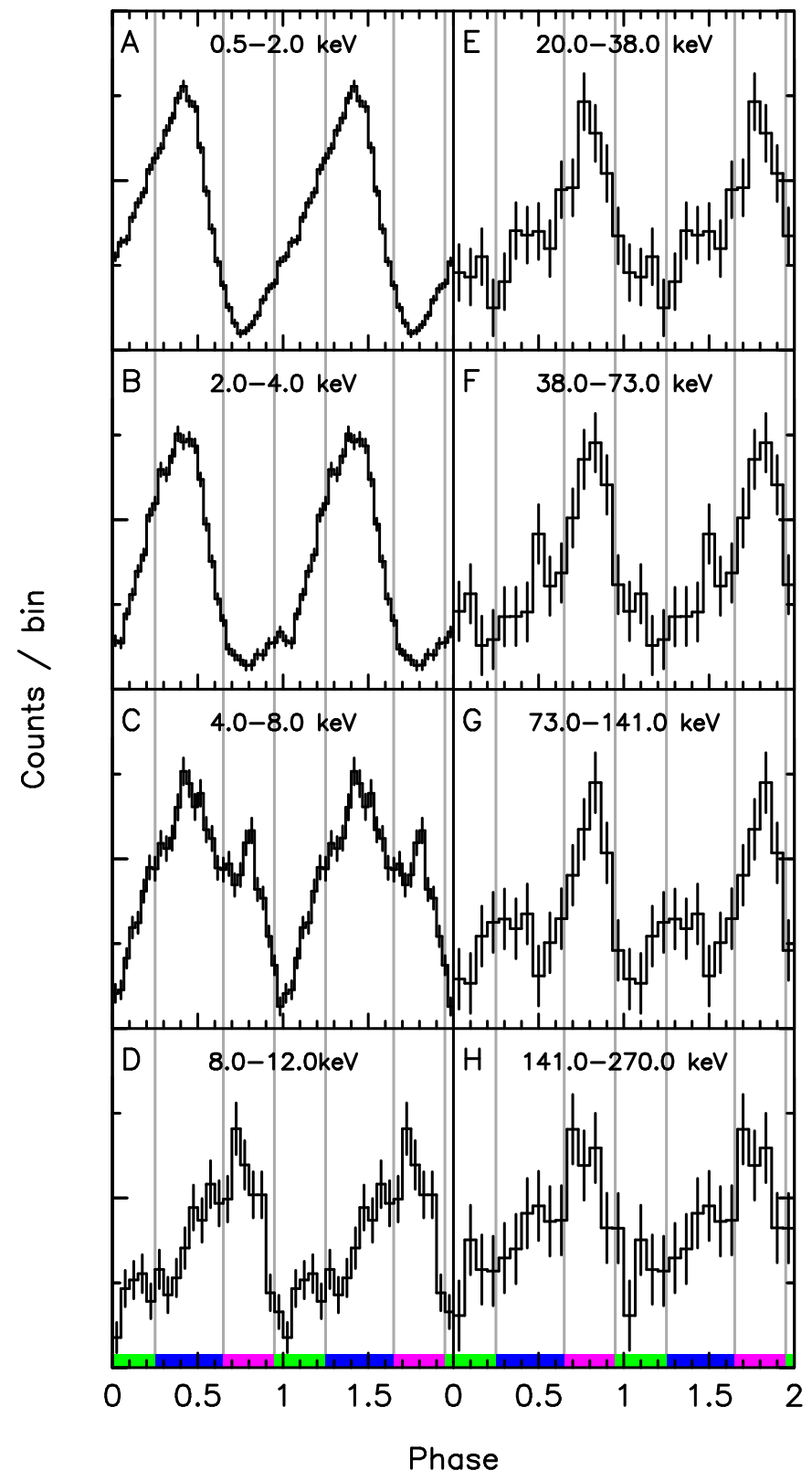

Fig. 6. Pulse profiles from soft to hard X-rays. XMM-Newton pulse profiles in the energy range $0.5-12.0 \mathrm{keV}$ are shown in panels A, B, C and D. Panels E, F, G and $\mathrm{H}$ show INTEGRAL pulse profiles in the energy range $20-270 \mathrm{keV}$. The differential energy ranges are indicated in the figures. The phase intervals are indicated as in Fig. 5 .

proved pulse profile with a $12.3 \sigma$ detection significance $\left(\mathrm{Z}_{3}^{2}\right.$ test; Buccheri et al. 1983) for energies 20-270 keV (Fig. 5). The profile shows a single pulse which peaks around phase 0.8 with a steep trailing wing dropping off to the DC level at phase $\sim 1.05$ (0.05). On the leading wing there appears to be a weak pulse or shoulder.

Presented in Fig. 66E-H are four exponentially-binned differential INTEGRAL pulse profiles. The profiles have significances of $6.3 \sigma, 6.8 \sigma, 6.2 \sigma$ and $3.5 \sigma$, respectively, using a $\mathrm{Z}_{2}^{2}$ test. All profiles show single pulses which all peak at phase $\sim 0.8$, like for the total pulse in Fig.5.

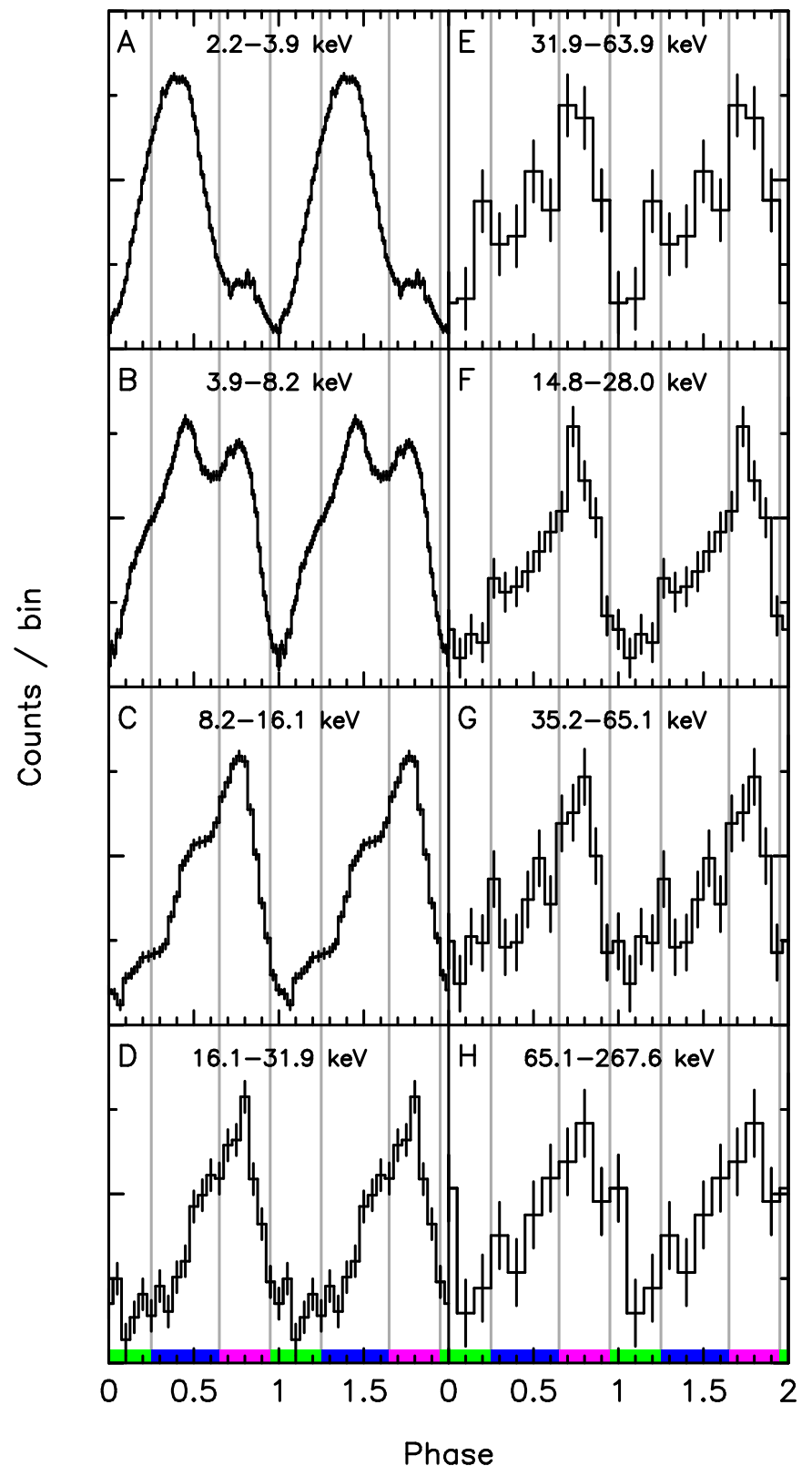

Fig. 7. Pulse profiles from RXTE. RXTE-PCA pulse profiles in the energy range $2.2-63.9 \mathrm{keV}$ are shown in panels $\mathrm{A}, \mathrm{B}, \mathrm{C}$, D and E. RXTE-HEXTE pulse profiles are shown in panels F, $\mathrm{G}$ and $\mathrm{H}$ within the energy range $14.8-267.6 \mathrm{keV}$. The phase intervals are indicated as in Fig. 5.

The complementary high-statistics XMM-Newton pulse profiles at lower energies $(0.5-12 \mathrm{keV})$ are shown in Fig. 6]D. The pulse profile with the lowest statistics, in panel $\mathrm{D}$, still has a significance of $10.7 \sigma\left(\mathrm{Z}_{3}^{2}\right.$ test $)$. The drastic change in morphology moving up in energy from $0.5 \mathrm{keV}$ up to $270 \mathrm{keV}$ is evident and will be investigated further. Note that in the timing analysis the pulsed emission has been detected to higher energies than the total emission in the sky maps. 


\subsubsection{RXTE-PCA and RXTE-HEXTE pulse profiles}

The RXTE-PCA bridges the observational gap (12-20 keV) in energy between XMM-Newton and INTEGRAL, with XMMNewton being sensitive down to $\sim 0.5 \mathrm{keV}$ and INTEGRAL extending the coverage to $\sim 300 \mathrm{keV}$. In addition, RXTE-HEXTE is sensitive over about the same energy band as INTEGRALISGRI. Analysis of data from these three missions allow for consistency checks, particularly when the observations are covering the same epochs and for checks on long-term variability. RXTE observations set A (Table 3) covers about 6 years (mostly before the launch of INTEGRAL) and was used by Kuiper et al. (2006). Set B overlaps in time with the INTEGRAL observations up to December 2006. Therefore, we first compared the time-averaged RXTE-PCA pulse profiles in 5 differential energy bands for set $\mathrm{A}$ with those for set $\mathrm{B}$. We did not find significant differences in these time averaged (over years) profile shapes. This is in agreement with the findings by Dib et al. (2008), who compared the profile shapes for seven (glitch-free) intervals of the total set A plus B. We should note that the PCA gain changed over time, what can offer an explanation for some indications for small changes with time in the profiles as discussed in Dib et al. (2008).

In order to exploit the maximal statistics, we created pulse profiles using all available data collected with the PCA and HEXTE over nine years (see Table 3 and Sect. 2.2). Before summing the profiles obtained at different epochs we corrected for the above-mentioned PCA gain drift in the conversion from channels to energy. In Fig. 7the RXTE-PCA (panels A-E) and HEXTE (panels F-H) pulse profiles are presented. The 'lowestsignificance' profiles above $\sim 15 \mathrm{keV}$ (Fig.7D-H) have significances of $18.5 \sigma, 4.8 \sigma, 12.0 \sigma, 6.4 \sigma$ and $4.3 \sigma$, respectively.

\subsubsection{Pulse profile changes with energy}

Comparing the profile shapes derived with INTEGRAL (Fig.6) with those obtained with the PCA and HEXTE in similar energy bands (Fig. 7), shows very good agreement, INTEGRAL achieving the best statistics above $70 \mathrm{keV}$. Above $\sim 20 \mathrm{keV}$ there does not seem to occur significant changes in morphology with energy. The pulse profiles are statistically similar which was tested using a combination of a Pearson $\chi^{2}$ (see e.g. Sect. 11.2 of Eadie et al. 1971) and a Run test (see e.g. Sect. 11.3.1 of Eadie et al. 1971).

Below $20 \mathrm{keV}$, the situation is different: The XMM-Newton and RXTE-PCA profiles show strong morphology changes as a function of energy. This has been noted in a number of earlier publications (e.g. Sugizaki et al. 1997; Israel et al. 2001; Kuiper et al. 2006). In fact, the morphology is changing faster with energy than can be seen in the broad energy bins selected for Fig. 6 and Fig. 7. In addition, there are systematic effects which should be realized when comparing XMMNewton and PCA profiles. For example, in the measured energy band 2.2-3.9 keV (Fig. 7A) one can see in the PCA profile a small secondary peak at phase $\sim 0.8$, which is absent in the corresponding XMM-Newton profile (Fig. 6B). This can be explained with the significantly coarser energy resolution of the PCA, accepting more events with photon energy above 4
$\mathrm{keV}$ in the band with measured energies below $4 \mathrm{keV}$. For the same reason, this secondary peak at phase 0.8 in the PCA 3.98.2 profile (Fig. 7B) is higher than found in the corresponding XMM-Newton profile (Fig.6C), because this pulse component is maximal for energies above $8 \mathrm{keV}$. Therefore, a more accurate comparison can be made between the pulse characteristics measured with XMM-Newton and the RXTE-PCA by performing phase-resolved spectroscopy in which the energy resolution is automatically taken into account (addressed in Sect. 3.3.2).

Nevertheless, the XMM-Newton and RXTE-PCA profiles consistently show that the soft X-ray pulse has two components which peak at phases $\sim 0.4$ and $\sim 0.8$. The pulse peaking at phase $\sim 0.4$ is spectrally softer than the pulse peaking at phase $\sim 0.8$. As mentioned above, the latter is not visible in Fig. $6 \mathrm{~A}$ and $\mathrm{B}$. This peak starts contributing above $\sim 4 \mathrm{keV}$ (Fig. 6] C and Fig. 7 B) and is the dominating pulse component already above $\sim 8 \mathrm{keV}$ up to and including the hard X-ray band.

The soft pulse (peak at phase $\sim 0.4$ ) is gradually decreasing in strength as a function of energy and seems to disappear entirely at hard X-rays. Finally, there is an indication for a third pulse component, visible in the XMM profile below $2 \mathrm{keV}$ where the RXTE-PCA has no sensitivity. This component at phase $\sim 1.0$ fills up the soft pulse to a saw-tooth-like profile (Fig.6A), at least, there is a sudden, significant change in pulse shape in comparison to the profile above $2 \mathrm{keV}$.

\subsection{Energy spectra of the pulsed emission}

For INTEGRAL-ISGRI, XMM-Newton-PN, RXTE-PCA and RXTE-HEXTE pulsed spectra were created by analyzing the pulse profiles following the procedure described in Sect. 2. In Fig. 5 an example is given of how the pulsed counts are extracted as excess counts above a flat background level. In this case for 1RXS J1708-40, the $\sim 40 \%$ pulsed emission in the total INTEGRAL energy band is separated from the $\sim 60 \%$ DC component. For INTEGRAL pulse profiles for seven exponentially binned energy intervals within 20-300 keV were created. For XMM-Newton, RXTE-PCA and HEXTE pulse profiles in $20(0.5-12 \mathrm{keV}), 15(2.7-32.1 \mathrm{keV})$ and $5(14.8-267.5 \mathrm{keV})$ energy intervals were produced, respectively. In this section we first show the total-pulsed phase-averaged spectra. Then we show for all three missions the results of phase-resolved spectroscopy applied for three broad phase bins selected in the INTEGRAL profile in Fig. 5. Finally, we show phase-resolved pulsed spectra for narrow $(\Delta \phi=0.1)$ phase bins for RXTEPCA and INTEGRAL leading to the identification of three spectrally different components which contribute to the total pulsed emission.

\subsubsection{Total-pulsed spectra}

In Fig. 4 the time-averaged total-pulsed spectra are shown for all four instruments using all available exposures. The flux values from XMM-Newton, RXTE-PCA, RXTE-HEXTE and INTEGRAL are very consistent, yielding a continuous broadband spectrum from $0.5 \mathrm{keV}$ up to almost $300 \mathrm{keV}$. The RXTEPCA total-pulsed spectrum smoothly bridges the energy gap 
between INTEGRAL and XMM-Newton. The RXTE-HEXTE spectrum is in agreement with the INTEGRAL spectrum and also connects to the RXTE-PCA spectrum.

For RXTE-PCA we first created spectra for sets A and B separately, to verify whether the time-averaged 1RXS J170840 spectra differ in the years before and during the INTEGRAL observations. Within the statistical errors the spectra appeared to be identical, in flux and shape. This is in agreement with the recent findings of Dib et al. (2008), who also concluded that the pulsed emission is very stable over the whole time span from 1998 until 2006. Therefore, the combined spectrum with maximal statistics using both sets of data is shown in Fig. (4) and is further described below.

Fitting INTEGRAL, RXTE-PCA and HEXTE simultaneously with two power-law components yields an excellent fit for energies above $2.8 \mathrm{keV}$ (see Table 5). The soft power law has a photon index $\Gamma=2.79 \pm 0.07$ and the hard power law has a photon index $\Gamma=0.86 \pm 0.16\left(\chi_{r}^{2}=0.51\right.$, dof $\left.=22\right)$. Fitting the INTEGRAL spectrum separately it can be described by a single power-law model with a photon index $\Gamma=0.98 \pm 0.31\left(\chi_{r}^{2}\right.$ $=0.70$, dof $=5$ ), which is nicely within errors of the combined INTEGRAL-RXTE fit.

To determine the model parameters of the $0.5-12.0 \mathrm{keV}$ XMM-Newton spectrum the contribution of the hard powerlaw spectral component (significant above $\sim 5 \mathrm{keV}$ ) were added with fixed parameters (as determined from the INTEGRALRXTE fit) to the model and thereby effectively subtracted. Fitting the 0.5-12.0 keV XMM-Newton spectrum with a power-law yields a rather poor fit with photon index $\Gamma=$ $3.08 \pm 0.03\left(\chi_{r}^{2}=1.62\right.$, dof $\left.=15\right)$. This photon index is slightly softer than found for RXTE-PCA. However, the XMM-Newton spectrum in Fig. 44shows a discontinuity between 2 and $3 \mathrm{keV}$, just below the RXTE-PCA energy band. Phase-resolved spectroscopy in the next section will show that this discontinuity in the total-pulsed spectrum between 2 and $3 \mathrm{keV}$ is genuine. Indeed, for energies above $2.8 \mathrm{keV}$ an excellent fit can be obtained with a power law with a photon index $\Gamma=2.87 \pm 0.12$, which is in agreement with the RXTE-PCA spectrum above $2.8 \mathrm{keV}$ (in flux and index, see Table 5). The normalization of the XMM-Newton spectrum below $2.8 \mathrm{keV}$ is higher than the one above $2.8 \mathrm{keV}$, but the spectral shape is the same with an photon index $\Gamma=2.89 \pm 0.06$.

The XMM-Newton results imply an average pulsed fraction (defined as the pulsed emission divided by the total emission) of $(35.6 \pm 1.4) \%$ for the $2-10 \mathrm{keV}$ band. The INTEGRALRXTE fit gives a $20-150 \mathrm{keV}$ pulsed flux of $(2.56 \pm 0.40) \times$ $10^{-11} \mathrm{erg} \mathrm{cm}^{-2} \mathrm{~s}^{-1}$, resulting in an average pulsed fraction of $(39 \pm 6) \%$. The broad-band $2-10 \mathrm{keV}$ and $20-150 \mathrm{keV}$ pulsed fractions suggest a fairly constant pulsed fraction over the total high-energy window. However, in Fig. 4 it can be seen that the measured pulsed flux (204-300 keV) is on the extrapolation of the power-law fit to the total spectrum. At these high energies the pulsed fraction could be as high as $100 \%$.
Table 5. Spectral-fit parameters of the total-pulsed INTEGRAL, XMM-Newton, RXTE-PCA and RXTE-HEXTE fits (see Sect. 3.3.1). The $N_{\mathrm{H}}$ is fixed to $1.47 \times 10^{22} \mathrm{~cm}^{-2}$. The integrated fluxes are in units erg cm $\mathrm{cm}^{-2} \mathrm{~s}^{-1} \cdot F_{0}$ is the normalization at $1 \mathrm{keV}$ in units $\mathrm{ph} \mathrm{cm}^{-2} \mathrm{~s}^{-1} \mathrm{keV}^{-1}$. The subscripts $\mathrm{s}$ and $\mathrm{h}$ stand for 'soft' and 'hard' in the combined fit.

\begin{tabular}{|c|c|}
\hline \multicolumn{2}{|c|}{ INTEGRAL 20-270 keV } \\
\hline $\begin{array}{l}\Gamma_{\text {INTEGRAL }} \\
F_{20-150 \mathrm{keV}}\end{array}$ & $\begin{array}{c}0.98 \pm 0.31 \\
(2.53 \pm 0.40) \times 10^{-11}\end{array}$ \\
\hline $\begin{array}{l}\chi_{r}^{2} \quad(\text { dof }) \\
F_{20-270 \mathrm{keV}}\end{array}$ & $\begin{array}{c}0.70(5) \\
(4.89 \pm 1.12) \times 10^{-11}\end{array}$ \\
\hline \multicolumn{2}{|c|}{$\underline{\mathrm{XMM}-N \text { ewton } 0.5-2.8 \mathrm{keV}^{\dagger}}$} \\
\hline $\begin{array}{l}\Gamma_{\mathrm{XMM}-\text { Newton }} \\
F_{0 \mathrm{XMM}-\text { Newton }}\end{array}$ & $\begin{array}{c}2.89 \pm 0.06 \\
(1.95 \pm 0.07) \times 10^{-2}\end{array}$ \\
\hline $\begin{array}{l}\chi_{r}^{2} \quad(\mathrm{dof}) \\
F_{0.5-2 \mathrm{keV}}^{\dagger}\end{array}$ & $\begin{array}{c}0.46(7) \\
(4.69 \pm 0.24) \times 10^{-11}\end{array}$ \\
\hline \multicolumn{2}{|c|}{$\underline{\text { XMM-Newton } 2.8-12 \mathrm{keV}^{\dagger}}$} \\
\hline $\begin{array}{l}\Gamma_{\mathrm{XMM}-\text { Newton }} \\
F_{0 \mathrm{XMM}-\text { Newton }}\end{array}$ & $\begin{array}{c}2.87 \pm 0.12 \\
(1.43 \pm 0.25) \times 10^{-2}\end{array}$ \\
\hline $\begin{array}{l}\chi_{r}^{2} \quad(\text { dof }) \\
F_{2-10 \mathrm{keV}}^{\dagger} \\
\text { RXTE-PCA+ } \\
\end{array}$ & $\begin{array}{c}0.39(6) \\
(1.21 \pm 0.05) \times 10^{-11} \\
\text { E+INTEGRAL } 2.8-270 \mathrm{keV}\end{array}$ \\
\hline $\begin{array}{l}\Gamma_{\mathrm{s}} \\
F_{0 \mathrm{~s}} \\
\Gamma_{\mathrm{h}} \\
F_{\mathrm{h}, 20-150 \mathrm{keV}}\end{array}$ & $\begin{array}{c}2.79 \pm 0.07 \\
(1.34 \pm 0.11) \times 10^{-2} \\
0.86 \pm 0.16 \\
(2.40 \pm 0.32) \times 10^{-11}\end{array}$ \\
\hline $\begin{array}{l}\chi_{r}^{2} \quad(\mathrm{dof}) \\
F_{2-10 \mathrm{keV}} \\
F_{10-20 \mathrm{keV}} \\
F_{20-150 \mathrm{keV}} \\
F_{20-270 \mathrm{keV}}\end{array}$ & $\begin{array}{c}0.51(22) \\
(1.243 \pm 0.011) \times 10^{-11} \\
(0.333 \pm 0.010) \times 10^{-11} \\
(2.60 \pm 0.35) \times 10^{-11} \\
(5.16 \pm 1.06) \times 10^{-11}\end{array}$ \\
\hline
\end{tabular}

$\dagger$ : Model includes fixed parameters $\Gamma_{\mathrm{h}}$ and $F_{\mathrm{h}, 20-150 \mathrm{keV}}$ of the combined RXTE-INTEGRAL spectrum to correct for the contribution of the hard X-ray spectrum in the XMM-Newton band. The integrated flux is the model flux including the hard component.

\subsubsection{Phase-resolved pulsed spectra}

The morphology of the 20-270 keV INTEGRAL pulse profile (Fig. 5) was used to define three broad phase intervals: $\mathrm{Ph} \mathrm{I}$ contains the DC level (green coloured in figures); Ph II contains the shoulder in the INTEGRAL band (blue coloured in figures); and Ph III contains the main pulse in the INTEGRAL band (magenta coloured in figures). The phase interval boundaries are listed in Table 6 and are indicated with vertical lines and colour bars in Figs. 5, 6 and 7 In the latter figures is also visible how these phase intervals relate to the profile shapes at energies below $10 \mathrm{keV}$.

In the upper panels of Fig. 8 the resulting pulsed-emission spectra for these three phase intervals are shown, in each case together with the total-pulsed spectrum, and for XMM-Newton and INTEGRAL also with the total-emission spectra; XMMNewton in the upper-left panel; RXTE-PCA in the top-center panel; and in the top-right panel XMM-Newton, RXTE-PCA and INTEGRAL together. In the RXTE-PCA analysis we have 

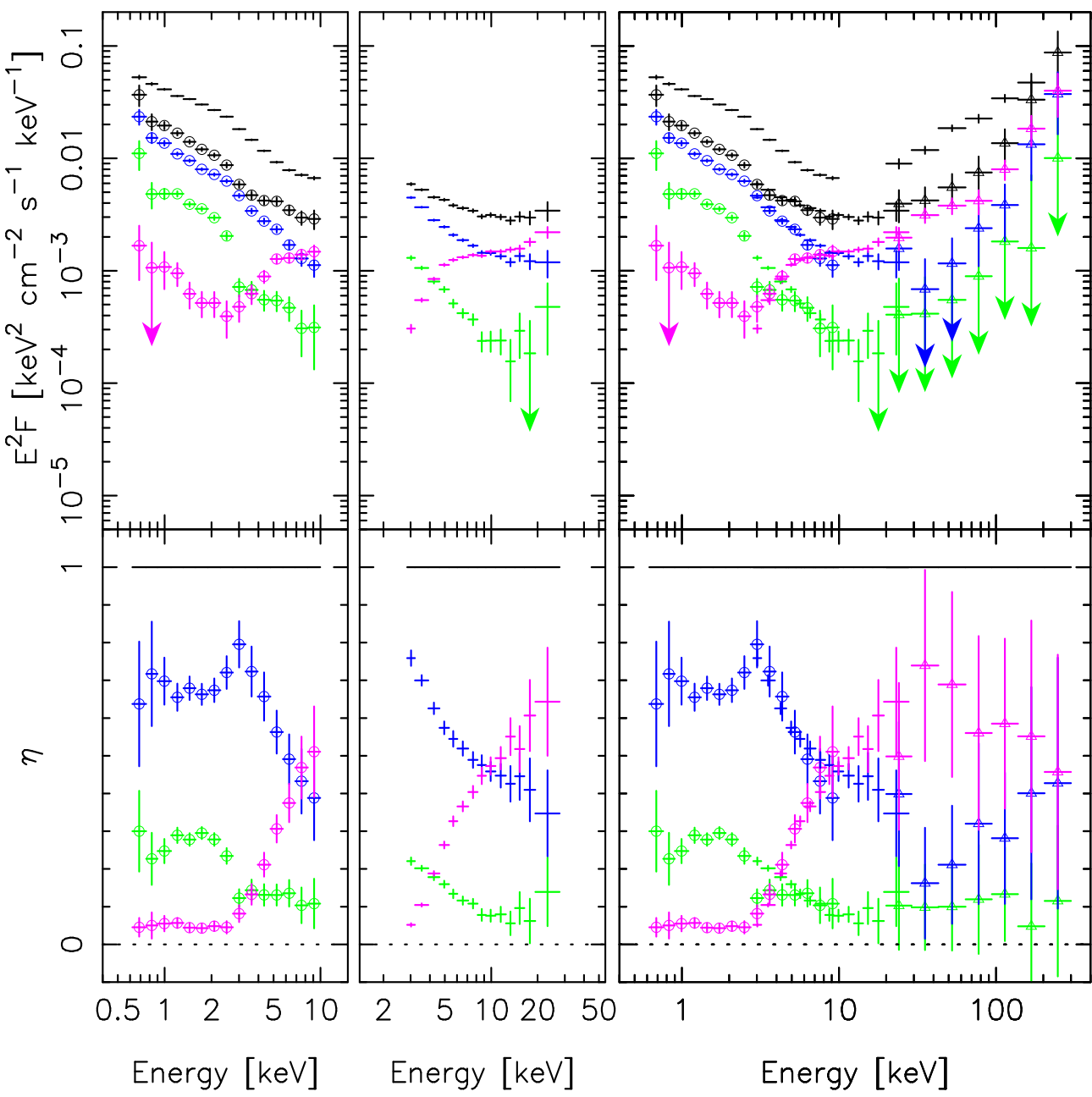

Fig. 8. Phase-resolved results for XMM-Newton and RXTE-PCA are presented in the left and center panels, respectively. These data and the INTEGRAL (overlapping) results are shown together in the right panels. Total (except for RXTE), total-pulsed and phase-resolved pulsed spectra are presented in the top panels. The total spectra and total-pulsed spectra are plotted in black. The phase resolved spectra for Ph I, Ph II and Ph III (see Table 6) are plotted in green, blue and magenta, respectively. The XMMNewton pulsed spectra are also indicated with open circle symbols while the INTEGRAL pulsed spectra are indicated with a triangle symbols. The data points with arrows indicate that the flux values have significances less than $1.5 \sigma$ and the positive $1 \sigma$ error is drawn. In the bottom panels $\eta$ - defined as the fraction of the total pulsed emission within a phase interval - is shown in the same colour scheme as the spectra.

Table 6. Selected phase intervals for extraction of high-energy spectra, using the pulse-shape morphology of the INTEGRALIBIS-ISGRI 20-270 keV pulse profile (Fig. 5).

\begin{tabular}{lll}
\hline \hline & Phase interval & Component \\
\hline $\mathrm{Ph}$ I & {$[0.95,0.25\rangle$} & DC level INTEGRAL profile \\
$\mathrm{Ph}$ II & {$[0.25,0.65\rangle$} & Shoulder in INTEGRAL band \\
$\mathrm{Ph}$ III & {$[0.65,0.95\rangle$} & Main hard INTEGRAL pulse \\
\hline
\end{tabular}

again verified that the three phase-resolved spectra for Set A and Set B are fully consistent: there is no sign for long-term variability in shape and flux down to the 5\%-level.

We first focus on the pulsed-emission spectrum of $\mathrm{Ph}$ III which contains the main hard INTEGRAL pulse. This Ph III spectrum follows the total-pulsed spectrum in the INTEGRAL band, as expected. However, Ph III shows below $10 \mathrm{keV}$ a com- pletely different spectral behaviour with respect to the totalpulsed spectrum. Below $10 \mathrm{keV}$ where the total-pulsed spectrum turns up towards lower energies, this spectrum keeps going down steeply. The pulsed emission of Ph III reaches a minimum in luminosity around $\sim 2.5 \mathrm{keV}$. At lower energies it turns up again. This remarkably spectral shape is measured consistently with XMM-Newton and RXTE-PCA, as can be seen in the right panel of Fig. 8 (magenta coloured data points). Therefore, we can exclude a systematic problem as cause of this appearance. It is also remarkable how smoothly the combined XMM-Newton and nine-year-average RXTE-PCA spectra join the four-year-average INTEGRAL spectrum. This is strong evidence for a very stable configuration of the production sites of the high-energy emission in the magnetar magnetosphere over almost a decade.

It is obviously not straightforward to fit the spectrum of Ph III. We attempted to make an empirical description to 'quan- 
Table 7. Spectral-fit parameters for Ph III INTEGRAL, XMMNewton, RXTE-PCA and RXTE-HEXTE fits (see Sect. 3.3.2). The $N_{\mathrm{H}}$ is fixed to $1.47 \times 10^{22} \mathrm{~cm}^{-2} . k T$ and $E_{\text {break }}$ are in units $\mathrm{keV} . F_{0}$ is the normalization at $1 \mathrm{keV}$ in units $\mathrm{ph} \mathrm{cm}^{-2} \mathrm{~s}^{-1} \mathrm{keV}^{-1}$. The integrated fluxed are in units erg $\mathrm{cm}^{-2} \mathrm{~s}^{-1}$.

\begin{tabular}{|c|c|}
\hline \multicolumn{2}{|c|}{$\frac{\text { XMM-Newton+RXTE-PCA+HEXTE+ }}{\underline{\text { INTEGRAL } 0.5-270 \mathrm{keV}}}$} \\
\hline $\begin{array}{l}k T \\
F_{0 k T} \\
\Gamma_{\mathrm{I}} \\
E_{\text {break }} \\
\Gamma_{\text {II }} \\
F_{0} \\
\Gamma_{\text {III }} \\
F_{20-150 \mathrm{keV}}\end{array}$ & $\begin{array}{c}0.23 \pm 0.02 \\
(2.82 \pm 0.64) \times 10^{-5} \\
-0.55 \pm 0.14 \\
4.92 \pm 0.11 \\
1.77 \pm 0.09 \\
(2.05 \pm 0.52) \times 10^{-5} \\
0.02 \pm 0.50 \\
(0.86 \pm 0.22) \times 10^{-11}\end{array}$ \\
\hline $\begin{array}{l}\chi_{r}^{2}(\text { dof }) \\
F_{0.5-2 \mathrm{keV}}\left(\mathrm{erg} \mathrm{cm}^{-2} \mathrm{~s}^{-1}\right) \\
F_{2-10 \mathrm{keV}}\left(\mathrm{erg} \mathrm{cm}^{-2} \mathrm{~s}^{-1}\right) \\
F_{20-150 \mathrm{keV}}\left(\mathrm{erg} \mathrm{cm}^{-2} \mathrm{~s}^{-1}\right) \\
F_{20-270 \mathrm{keV}}\left(\mathrm{erg} \mathrm{cm}^{-2} \mathrm{~s}^{-1}\right)\end{array}$ & $\begin{array}{c}0.90(29) \\
(0.19 \pm 0.07) \times 10^{-11} \\
(0.23 \pm 0.07) \times 10^{-11} \\
(1.54 \pm 0.18) \times 10^{-11} \\
(3.74 \pm 0.65) \times 10^{-11}\end{array}$ \\
\hline \multicolumn{2}{|c|}{ INTEGRAL $20-270 \mathrm{keV}$} \\
\hline $\begin{array}{l}\Gamma_{\text {INTEGRAL }} \\
F_{20-150 \mathrm{keV}}\left(\mathrm{erg} \mathrm{cm}^{-2} \mathrm{~s}^{-1}\right)\end{array}$ & $\begin{array}{c}1.09 \pm 0.16 \\
(1.53 \pm 0.12) \times 10^{-11}\end{array}$ \\
\hline $\begin{array}{l}\chi_{r}^{2}(\text { dof }) \\
F_{20-270 \mathrm{keV}}\left(\mathrm{erg} \mathrm{cm}^{-2} \mathrm{~s}^{-1}\right)\end{array}$ & $\begin{array}{c}1.15(5) \\
(2.81 \pm 0.42) \times 10^{-11} \\
\end{array}$ \\
\hline
\end{tabular}

tify' the discontinuities using all data points from INTEGRAL, RXTE-PCA and XMM-Newton. We succeeded in describing the spectrum over the whole $0.5-270 \mathrm{keV}$ band with a black body and three power-law segments (i.e. one broken power law plus a power law; $\chi_{r}^{2}=0.90 \mathrm{dof}=29$, see Table 7). The total fit contains a soft thermal black-body component below $2 \mathrm{keV}$ $(k T=0.23 \pm 0.02 \mathrm{keV})$. This component can be due to a tail of the soft pulse which peaks in Ph II. Above $\sim 2 \mathrm{keV}$ the spectrum drastically hardens. The spectrum up to the break energy $E_{\text {break }}=4.92 \pm 0.11 \mathrm{keV}$ is extremely hard with a photon in$\operatorname{dex} \Gamma=-0.55 \pm 0.14$. After the break a softer power law is required with photon index $\Gamma=1.77 \pm 0.09$. Finally, in order to account for the harder INTEGRAL spectrum a third power law is required with photon index $\Gamma=0.02 \pm 0.50$, which is dominating above $\sim 60 \mathrm{keV}$. It is obvious that very different production processes contribute in this phase interval ( $\mathrm{Ph}$ III) to the high-energy (pulsed) spectrum.

Fitting only the INTEGRAL spectrum with a power law, a photon index $\Gamma=1.09 \pm 0.16$ gives the best description. (Table7).

Also the spectral shape of the pulsed emission in $\mathrm{Ph}$ II (centered on the soft pulse peaking at phase $\sim 0.4$, blue coloured in figures) is very different from that of the total-pulsed spectrum.

The independent XMM-Newton and RXTE-PCA spectra are again fully consistent. A broad soft component below about $8 \mathrm{keV}$ turns in the RXTE band above $8 \mathrm{keV}$ into a component with power-law index $\sim 2$, but is not detected around 20 $\mathrm{keV}$. The flux measurements close to energies as high as 100 $\mathrm{keV}$ are likely not related to the softer X-ray components and can be explained as emission from a tail of the main hard-X-

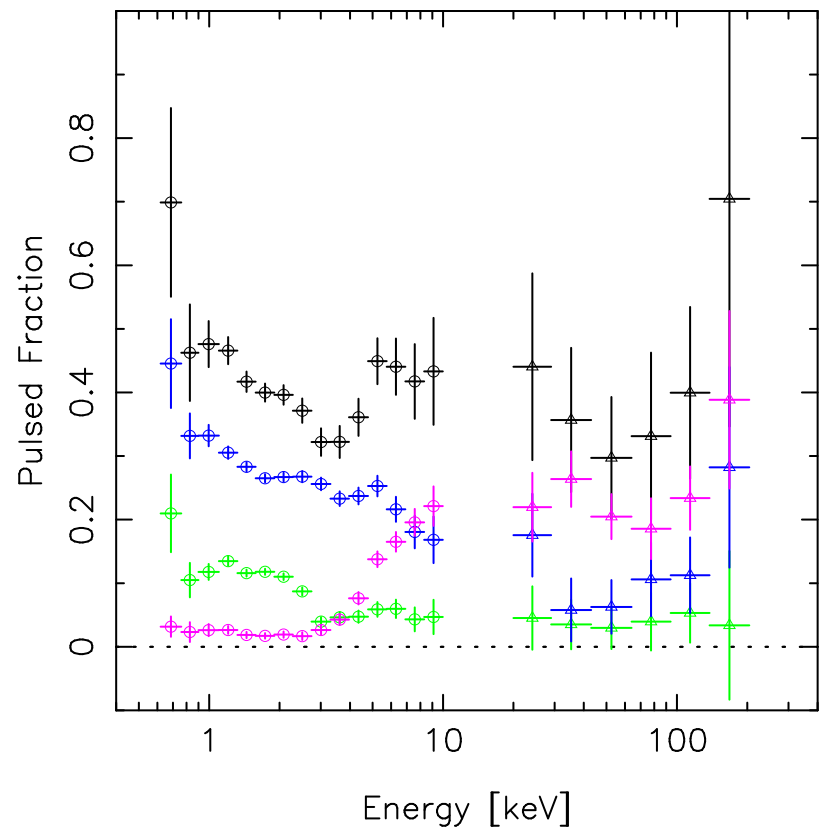

Fig. 9. The pulsed fractions of 1 RXS J1708-40 as a function of energy using XMM-Newton below $10 \mathrm{keV}$ and INTEGRAL above $20 \mathrm{keV}$. The total pulsed fraction is drawn in black and plotted in colour are the pulsed fractions for the three phase intervals (see Table 6).

ray INTEGRAL pulse in Ph III. No sensible model fits can be made to the total combined Ph II spectrum. A good fit can be obtained considering only XMM-Newton data, namely with a broken power-law with break energy $E_{\text {break }}=2.49 \pm 0.15 \mathrm{keV}$, consistent with the energy where in the total pulsed spectrum the discontinuity was found. Below the break energy the powerlaw model has a photon index $\Gamma=2.83 \pm 0.04$, above this energy $\Gamma=3.38 \pm 0.06$.

$\mathrm{Ph}$ I contains the left wing of the soft pulse below $8 \mathrm{keV}$, and harbours the DC level in the INTEGRAL band (green coloured in figures). Therefore, no significant flux is measured at hard X-rays. Also in this case no sensible model combination (black body, logparabola, power law) can describe the combined XMM-Newton-RXTE-PCA spectrum. Furthermore, the XMM-Newton spectrum exhibits again a drastic drop in flux between 2 and $3 \mathrm{keV}$. The fact that the RXTE-PCA data points between 2 and $3 \mathrm{keV}$ do not follow this sudden drop, might be explained with the coarser energy resolution of RXTE-PCA, combined with a strong gradient in the sensitive area and underlying photon spectrum.

A different way to study the relative contributions of the pulsed components in the three selected phase intervals as a function of energy is by plotting the $\eta$ parameter, defined as the fraction of the total-pulsed emission. It shows more clearly discontinuities in the dependence on energy. In the bottom panels of Fig. $8 \eta$ is presented for the different observations. The bottom panel for XMM-Newton shows that the Ph III contribution to the pulsed emission is a few percent up to $\sim 2.5 \mathrm{keV}$. Above $2.5 \mathrm{keV}$ the contribution increases as a function of energy to $\sim 50 \%$ at $10 \mathrm{keV}$. This picture is fully confirmed by RXTE, which shows that $\eta$ is still increasing above $10 \mathrm{keV}$ up 


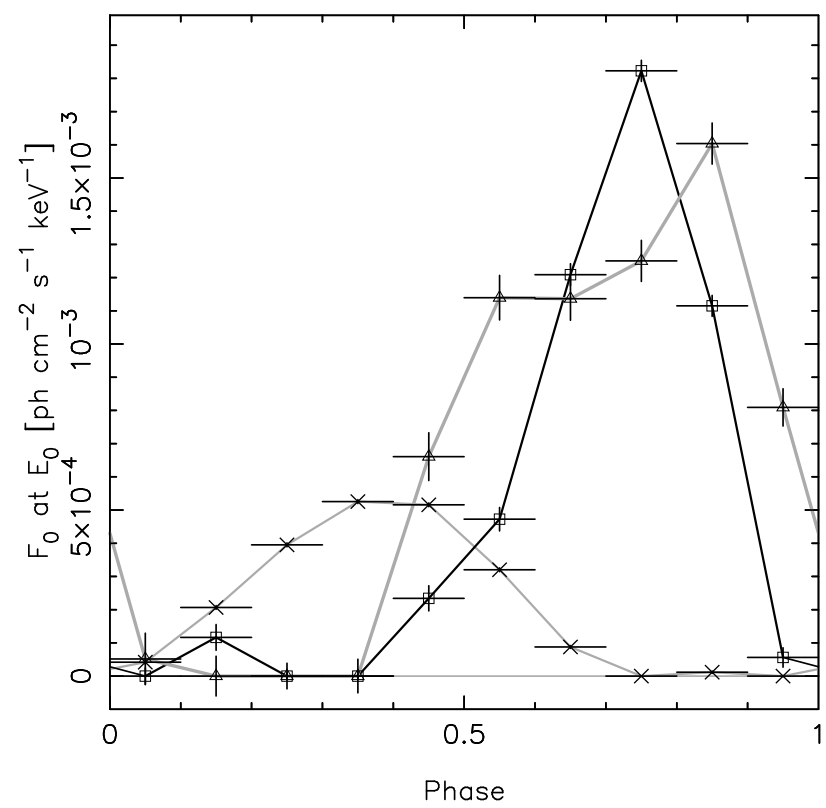

Fig. 11. Phase distributions of the normalizations $F_{0}$ of the three spectral-model components (see Table 8) taken at pivot energy $E_{0}=15.0433 \mathrm{keV}$ for the 10 narrow phase-interval spectra. Indicated with crosses and a thin grey line are the normalizations of the soft power-law component, with triangles and thick grey line the hard power-law component and with squares and a black line the composite spectral component.

to $\sim 60 \%$ around $30 \mathrm{keV}$ and stabilizes around this value in the INTEGRAL band.

The opposite can be seen for the contribution to the pulsed flux from Ph II. A high $\eta$ of $\sim 70 \%$, fairly constant up to $\sim 3 \mathrm{keV}$ from where the contribution abruptly decreases to $\sim 40 \%$ at $10 \mathrm{keV}$. Also this decline is observed with RXTE. The behaviour in the INTEGRAL band is not clear. Finally, $\mathrm{Ph}$ I shows a somewhat similar trend as $\mathrm{Ph}$ II with a significant contribution below $\sim 3 \mathrm{keV}$ of $\sim 28 \%$, which decreases to $\sim 8 \%$ above $\sim 8 \mathrm{keV}$ and only upper limits in the INTEGRAL band.

For XMM-Newton and INTEGRAL the pulsed fraction is shown in Fig. 9. The pulsed fraction (total-pulsed emission divided by total emission) of 1RXS J1708-40 is around $40 \%$ from soft to hard X-rays (see also Sect.3.3.1). However, we can note here, that the pulsed fraction is not constant with energy, but shows significant variations with energy below $10 \mathrm{keV}$. In the INTEGRAL band the error bars are too large to draw firm conclusions. In Fig. 9 the pulsed fractions for the three phase intervals are also shown (i.e. emission in $\mathrm{Ph} \mathrm{I}$, II or III divided by total emission). As was also clear from the dependence of $\eta$ on energy in Fig. 8, the contribution of the hard X-ray pulse increases with energy above $\sim 3 \mathrm{keV}$, while the pulsed fractions of the other two phase intervals are decreasing with energy.

\subsubsection{Narrow-band phase resolved pulsed spectra from RXTE}

The phase-resolved spectroscopy presented in Sect. 3.3.2, selecting three broad phase intervals, unambiguously revealed that spectrally different components contribute to the total pulsed emission of 1RXS J1708-40. A similar conclusion was earlier reached for AXP 4U 0142+61 (den Hartog et al. 2008). However, the situation appears to be much more complex for 1RXS J1708-40. The XMM-Newton spectra below $\sim 2 \mathrm{keV}$ suggest a soft thermal component, which is not unexpected. However, this component can not be easily separated from components with apparently complex spectral shapes contributing to the total pulsed emission above $2 \mathrm{keV}$. Therefore, we exploited the high statistics of the total $\sim 600 \mathrm{ks}$ of RXTEPCA data to explore in more detail the spectral characteristics as a function of phase above $2 \mathrm{keV}$ and the connection to the INTEGRAL band. We repeated the phase-resolved spectroscopy but now for 10 narrow bins each covering 0.1 in phase. Applying the method described in Sect. 2.2 the 10 phaseresolved spectra have been extracted and are shown in Fig. 10 .

The variations in spectral shape with phase are drastic. In the first 4 phase bins between phase 0.0 and 0.4 , we see a spectral tail of the soft component peaking in energy in the XMMNewton band below $1 \mathrm{keV}$. These spectra can be accurately fitted for energies above $2.8 \mathrm{keV}$ with a single power-law model. The index for the best fit to the sum of the spectra between phase 0.0 and 0.3 is $\Gamma=3.54 \pm 0.01$. In the phase bins $0.4-$ 0.5 and $0.5-0.6$ a significant hardening of the spectra sets in for energies between about $5 \mathrm{keV}$ and $20 \mathrm{keV}$. In the following phase bins $0.6-0.7$ and $0.7-0.8$, getting closer to the phase of the main INTEGRAL pulse, evidence is visible for the presence of the hard power-law component with index $\sim 1.0$ above $20 \mathrm{keV}$. This latter hard power-law spectrum is dominating the energy budget for the last three phase bins. More surprisingly, in the two phase windows between 0.7 and 0.9 , centered on the INTEGRAL main pulse, a very hard part of the spectrum is apparent in a narrow energy interval above $\sim 2 \mathrm{keV}$. This is most extreme for the phase interval $0.7-0.8$ with a 'local' photon in$\operatorname{dex} \Gamma=-1.49 \pm 0.21$ (notice the sign!) up to a break energy $E_{\text {break }}=4.69 \pm 0.04$. Finally, the spectrum for phase interval 0.9-1.0, the last fraction of the trailing wing of the INTEGRAL main pulse, can be fitted with a single power law with best-fit photon index $\Gamma=0.99 \pm 0.05$. Moving only $10 \%$ in phase to the next phase interval $0.0-0.1$, the hard spectrum 'switches' and becomes again very soft with $\Gamma=3.58 \pm 0.34$.

From Fig. 10 it is evident that different spectral components contribute to the total pulsed emission and dominate in different phase intervals. Two components can clearly be identified: A soft power-law component with index $\Gamma \sim 3.6$ and a hard power-law component with index $\Gamma \sim 1.0$. Then there appears to be a third component, dominating in the energy band $\sim 3$ to $20 \mathrm{keV}$ for phases between 0.7 and 0.9 . A clear characteristic of this spectral component is a very steep rise above $2.3 \mathrm{keV}$ ( $\Gamma \sim-1.5)$. We investigated what shape such a third component should have in order to explain, together with two power-law components, all spectra in Fig. 10. This shape should give a clear constraint for any proposed production mechanism.

We followed the following procedure. The hard power-law component was adopted from the best fit to the spectrum for phase 0.9-1.0: $\Gamma=0.99$. Then we investigated empirically what spectral shape, together with the power-law model with $\Gamma=0.99$, can accurately describe the spectrum in phase inter- 


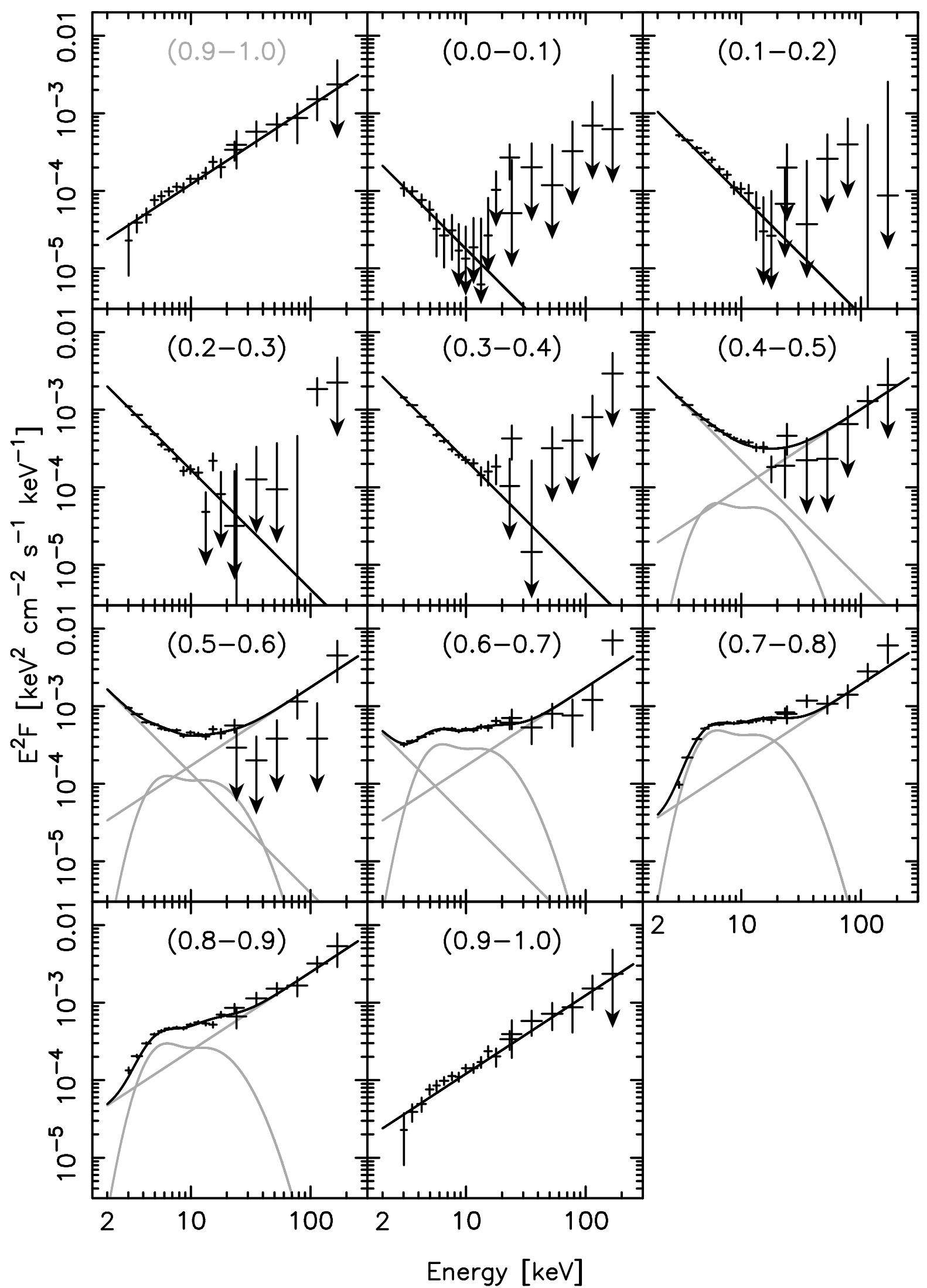

Fig. 10. Narrow-band phase-resolved spectroscopy of 1RXS J1708-40 using RXTE-PCA and INTEGRAL-ISGRI data. Each panel shows the spectrum of a phase interval with $\Delta \phi=0.1$. The phase intervals are indicated in the panels. The data points with arrows indicate that the flux values have significances less than $1.5 \sigma$. The positive $1 \sigma$ errors are drawn. Total spectral fits and the three components (when contributing significantly) are drawn in black and grey, respectively (See Sect 3.3.3 and Fig. 10). 
Table 8. Model parameters of the three spectral components that are used to fit the 10 narrow-band phase-resolved RXTEPCA and INTEGRAL-ISGRI spectra. The three components are a soft and a hard power-law component, and a curved component which is the sum of two logparabolic functions. For each component $F_{0}$ (in units $\mathrm{ph} \mathrm{cm}^{-2} \mathrm{~s}^{-1} \mathrm{keV}^{-1}$ ) is the normalization taken at the pivot energy $E_{0}=15.0433 \mathrm{keV}$ which is plotted as a function of phase in Fig. 11 .

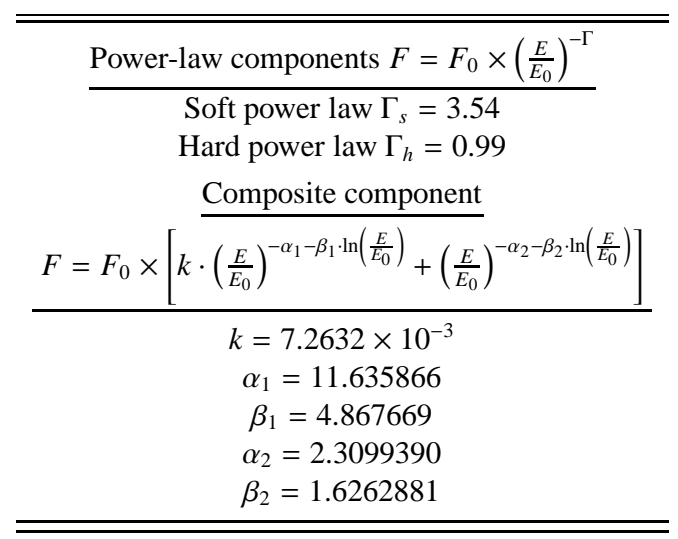

val 0.7-0.8. This turned out to be the sum of two logparabolic functions of which the parameters are given in Table 8 . Finally, for the index of the soft power-law model we adopted the best fit to the summed spectrum in the phase interval 0.0-0.3: $\Gamma=3.54$.

We now attempted to describe the measured spectral distributions in the 10 narrow phase intervals in terms of just these three model shapes with free normalizations. Interestingly, the resulting total fits are very satisfactory for all narrow phase intervals (see Fig. 10). The normalizations of the three models are shown in Fig. 11 Effectively, this figure shows the pulse profile of each spectral component contributing to the total-pulsed spectrum, i.e. a complete decoupling based on the measured spectral and timing characteristics. In Fig. 10 we show for all 10 spectra the contributions of the three spectral components to the model fit, but only when components were statistically required to reproduce the measured spectra. Fig. 11 together with Fig. 10 show now the characteristics of the three components which can reproduce together the total pulsed emission, the pulse shapes and their spectra. Furthermore, we have shown above that our results indicate that these characteristics are invariant over almost a decade of observations.

\section{Summary}

In this paper we have presented new and more detailed characteristics of 1RXS J1708-40 in the hard X-ray/soft gammaray regime $(>20 \mathrm{keV})$ using INTEGRAL-ISGRI and SPI data. Following the approach in our earlier paper on AXP 4U 0142+61 (den Hartog et al. 2008) we extended the energy window to lower energies to obtain a broader high-energy view using archival RXTE and XMM-Newton observations. The emphasis of this work on broad-band phase-resolved spectroscopy was aimed at the identification of distinctly different compo- nents contributing to the total-pulsed emission. What follows is a summary of the main results.

\subsection{Total high-energy emission above $20 \mathrm{keV}$ of 1RXS J1708-40}

1) Using all available INTEGRAL data taken with INTEGRAL ISGRI during 2003-2006 - adding up to 5.2 Ms effective onsource exposure - the time-averaged $20-175 \mathrm{keV}$ spectrum can be described by a power-law with photon index $\Gamma=1.13 \pm 0.06$. The luminosity is $1.34 \times 10^{35} \mathrm{erg} \mathrm{s}^{-1}(20-175 \mathrm{keV})$ adopting a distance of $3.8 \mathrm{kpc}$ (Sect. 3.1.2 Fig. 4 Table 4 ).

2) There is no indication for a spectral break in the INTEGRAL spectrum up to energies of $\sim 175 \mathrm{keV}$. Including in the spectral fit earlier published COMPTEL flux upper limits for energies above $750 \mathrm{keV}$ (Kuiper et al. 2006) and assuming a logparabolic shape for the spectrum above $20 \mathrm{keV}$, the maximum luminosity is found for an energy of $\sim 290 \mathrm{keV}$ (Sect. 3.1.2 Fig. (4).

\subsection{Long-term variability in the total emission of 1RXS J1708-40}

1) In the INTEGRAL data no significant long-term time variability for energies above $20 \mathrm{keV}$ is found on time scales of one year and half a year in total flux and spectral index. There is only one measurement with a $\sim 3 \sigma$ indication for a flux increase in nine observations. The total flux $(20-150 \mathrm{keV})$ and the spectral index are stable within $22 \%$ and $18 \%$ (both $1 \sigma$ ), respectivily (Sect. 3.1.1] Figures 12 2 $\mathcal{E}$ 3).

2) Our analysis of all available ISGRI data rejects the claim by Götz et al. (2007) of correlated variability between the hard $\mathrm{X}$-ray emission measured with ISGRI and soft X-ray emission from 1RXS J1708-40 in relation to a (candidate) glitch (Sect. 3.1.1 Fig. 3).

\subsection{Pulse profiles}

1) ISGRI measures significant pulse profiles for energies up to $270 \mathrm{keV}$ (Sect. 3.2.1] Figures 5 G 6 ).

2) High-energy pulse profiles measured with XMM-Newton, RXTE-PCA and HEXTE and INTEGRAL-ISGRI over the broad energy band $0.5 \mathrm{keV}$ up to $270 \mathrm{keV}$ are consistent in morphology when measured in the same differential energy bands, independent of the epoch of the observation; apparent differences are either due to statistics or due to differences in energy response (Sect. 3.2 Figures 6 G 7 ).

3) Fast morphology changes with energy are visible in the pulse profiles below $\sim 20 \mathrm{keV}$, (earlier noted by e.g. Sugizaki et al. 1997; Israel et al. 2001; Kuiper et al. 2006) In the INTEGRAL band the pulse profiles do not show morphology changes (Sect. 3.2. Figures 6 [ $\mathcal{E} 7$ 7).

4) Three different pulse components are recognized in the pulse profiles: a) A hard pulse peaking around phase 0.8 ; which starts contributing to the pulse profiles above $\sim 4 \mathrm{keV}, \mathrm{b}$ ) a softer pulse peaking around phase 0.4 ; which is not apparent at hard X-rays, c) a very soft pulse component below $2 \mathrm{keV}$ for which there is 
an indication around phase 1 where it fills the pulse profile to a saw-tooth-like shape (Sect. 3.2.1 Fig. 6).

\subsection{Total-pulsed spectra}

1) ISGRI measures the phase-averaged total-pulsed spectrum up to $270 \mathrm{keV}$ which can be described with a power law with photon index $\Gamma=0.98 \pm 0.31$ (Sect. 3.3.1. Fig. 4).

2) The average pulsed fraction in the INTEGRAL band is $39 \%$. Note, that the pulsed flux measured above $200 \mathrm{keV}$ is on the extrapolation of the total spectrum, consistent with being for $100 \%$ pulsed. Below $10 \mathrm{keV}$, the pulsed fraction as measured with XMM-Newton is not constant but varies between $\sim 31 \%$ and $\sim 48 \%$ in a complex way. (Sect. 3.3.1 Figures $4 \mathcal{G} 9$ ).

3) The total-pulsed spectrum from $0.5 \mathrm{keV}$ up to $270 \mathrm{keV}$ is very stable over time-scales of years. Firstly, the pulsedemission spectra (2.7-32.1 keV) of two sets of RXTEPCA data taken six years before and three years during the INTEGRAL operations, respectively, appeared to be statistically consistent within a few percent (see also Dib et al. 2008). Secondly, the summed RXTE-PCA total-pulsed spectrum joins smoothly that from XMM-Newton at lower energies, as well as those from RXTE-HEXTE and INTEGRAL at higher energies (Sect.3.3.1 Fig. 4).

4) Fitting INTEGRAL, RXTE-PCA and HEXTE simultaneously with two power-law components yields an excellent fit for energies above $2.8 \mathrm{keV}$ with a soft power-law model with photon index $\Gamma=2.79 \pm 0.07$ and a hard power-law model with photon index $\Gamma=0.86 \pm 0.16\left(\chi_{r}^{2}=0.51\right.$, dof $\left.=22\right)$. (Sect. 3.3.1 Table 5)

5) The XMM-Newton pulsed spectrum above $2.8 \mathrm{keV}$ is fully consistent in flux and power-law index with the corresponding RXTE-PCA spectrum, however, there is a discontinuity in the XMM-Newton spectrum, a drop in flux, between 2 and $3 \mathrm{keV}$ (Sect. 3.3.1. Fig. 4).

\subsection{Phase-resolved pulsed spectra}

1) The time-averaged phase-resolved spectra, selecting three broad phase intervals or narrow 0.1-phase-wide intervals, are connecting very smoothly over the total $0.5-300 \mathrm{keV}$ energy band from instrument to instrument: Another confirmation of the long-term many-year stability of the geometry and production mechanisms responsible for the emission (Sect. 3.3.2 Fig. $8 \mathcal{E}[10]$.

2) The XMM-Newton, RXTE-PCA and INTEGRAL phaseresolved spectra, selecting three broad phase intervals, have vastly different and complex spectral shapes, constituting together the total-pulsed spectrum (Sect. 3.3.2 Fig. 8).

3) $\mathrm{Ph}$ III (which contains the main hard INTEGRAL pulse) shows hardly any contribution to the pulsed spectrum for energies below $2.5 \mathrm{keV}$ (i.e. low $\eta$ ). This part of the spectrum can be described by a black-body with a temperature $k T$ of $0.23 \pm$ $0.02 \mathrm{keV}$. Above $2.5 \mathrm{keV}$, the energy where the Ph III pulsed emission reaches its minimum luminosity, the spectral shape becomes very complex: The spectrum first hardens dramatically (and $\eta$ increases) with a photon index $\Gamma=-0.55 \pm 0.14$ up to a break energy of $\sim 5 \mathrm{keV}$, consistently measured with XMM-Newton and RXTE-PCA. After the break the spectrum softens to a power-law with photon index $\Gamma=1.77 \pm 0.09$. In the INTEGRAL band it hardens again to a photon index $\Gamma=0.86 \pm 0.22$ (Sect. 3.3.2 Fig. 8 Table 7 .

4) The spectra of Ph I, DC-level in INTEGRAL pulse profile, and $\mathrm{Ph}$ II, 'shoulder' in INTEGRAL profile and main soft-Xray pulse, also appear to be complex. Both spectra are soft with maximum luminosity below $1 \mathrm{keV}$ and exhibit discontinuities around $2.8 \mathrm{keV}$, the energy where we noted a drop in flux in the total-pulsed spectrum of XMM-Newton. The emissions seem to vanish above $20 \mathrm{keV}$ (Sect. 3.3.2 Fig. 8).

5) Narrow-band phase-resolved spectroscopy in 10 phase bins reveals the cause of the complex behaviour of the emission spectra in the broader phase bins. The 10 spectra show gradual and sudden changes as a function of phase. The spectral complexity has become even clearer and is more extreme than seen in the broad-band phase-resolved spectra (Sect. 3.3.3 Fig. 10). 6) Each of the 10 pulsed spectra can be accurately described with a sum (free normalizations) of three components with very different spectral shapes: a) a soft power-law component with a photon index $\Gamma=3.54$, b) a hard power-law component with a photon index $\Gamma=0.99$, and c) a peculiar curved component which has been approximated by the sum of two logparabolic functions and contributes significantly between 4 and $20 \mathrm{keV}$ (Sect. 3.3.3 Fig. 10, Table 8).

7) The phase distributions of the normalizations to the three spectral components represent three decoupled pulse profiles which together constitute the total pulse profile. The soft component shows a single peak around phase 0.4 . The hard component shows a broad peak with a significant contribution from phase 0.4 to 1 . The curved component shows a narrower peak $(\sim 0.25$ in phase FWHM) which has its maximum in phase interval 0.7-0.8 (Sect. 3.3.3. Fig. 11).

\section{Discussion}

The detailed analysis performed in this work on the highenergy emission (0.5-300 keV) from 1RXS J1708-40 led to similar conclusions as were reached earlier for AXP 4U 0142+61 (den Hartog et al. 2008). Namely, that genuinely different pulse components with different spectra contribute to the total-pulsed emission. In the case of 1RXS J1708-40 not only the variation with energy of the structure of the pulse profiles could be used to identify these different components, similar to the case of $4 \mathrm{U} 0142+61$, but also the apparent fine structure in the phase-resolved spectra provided an efficient means to disentangle the pulsed emission into different components.

In den Hartog et al. (2008) we discussed the implications of the derived high-energy characteristics of $4 \mathrm{U} 0142+61$ for models developed in recent years for explaining the nonthermal, luminous hard X-ray emission from AXPs: 1) a quantum electro-dynamic model (QED) by Hevl \& Hernquist (2005a b) , 2) a corona model by Beloborodov \& Thompson (2007), further elaborated by Lyubarsky \& Eichler (2008) and 3) a resonant upscattering model by Baring \& Harding (2007). We concluded that all three models have difficulties in explaining many features of the detailed results (See the discussion in 
the paper by den Hartog et al. 2008). A confrontation of these models with our new results for 1RXS J1708-40 leads to similar conclusions. It is apparent that any attempt to explain the high-energy emission $(0.5-300 \mathrm{keV})$ should consider a stable (at least over a decade) three-dimensional geometry taking all relevant angles (the angle between the magnetic and the spin axis and the viewing angle) into account, as well as the physical production processes taking place on the surface of the neutron star and at different sites in the atmosphere and magnetosphere.

For 1RXS J1708-40 our results reveal a clear separation in phase, and therefore also of the production sites, of all pulsed hard X-ray emission above $20 \mathrm{keV}$ with photon index $\Gamma \sim 1.0$ from the pulsed soft thermal emission below $4 \mathrm{keV}$. This soft emission can be described with a black-body spectrum with $k T<0.4 \mathrm{keV}$ and a power-law spectral tail with photon in$\operatorname{dex} \Gamma \sim 3.5$. Such a clear separation is consistent with predictions from the QED model by Heyl (2007). However, the observed total and the pulsed hard X-ray spectra are harder (index $\sim 1.0$ ) than the photon index of 1.5 predicted by this model. Contrarily, the clear separation in phase between the thermal and non-thermal pulses makes an interpretation of the hard X-ray emission in terms of resonant magnetic upscattering of the thermal soft photons by ultra-relativistic electrons, accelerated along either open or closed magnetic field lines unlikely (proposed in the Compton-upscattering model by Baring \& Harding 2007). At least part of the thermal target photons should also arrive in the narrow phase interval of the non-thermal pulse. This problem might be circumvented if the thermal target photons are part of the isotropic thermal DC emission, and the thermal pulse at phase 0.4 is not related to the hard X-ray emission.

As mentioned before, Götz et al. (2007) reported the detection of correlated variability in hard X-ray flux and spectral index with similar correlated variations at low-energy X-rays from 1RXS J1708-40. This would imply correlated production processes at the same sites in the magnetosphere. However, our analysis disproved the claimed variability in the ISGRIdetected hard X-ray flux.

Very peculiar is the spectral shape of the component which contributes significantly to the total-pulsed emission from 1RXS J1708-40 between $4 \mathrm{keV}$ and $20 \mathrm{keV}$. It is not obvious what underlying emission processes can produce such a spectral shape. This component exhibits the most narrow pulse profile with a width of $\sim 0.25$ in pulsar phase (FWHM, see Fig. 11). It appears to be completely separated from the soft pulse, but it is centered in phase on the hard X-ray pulse which is about twice as broad. This means that the production site in the magnetosphere of this curved component is confined to a narrow region, possibly directed along open or the last closed magnetic-field lines, similar to different dipole scenarios proposed for radio pulsars (e.g. Ruderman \& Sutherland 1975; Usov \& Melrose 1995; Harding \& Muslimov 1998; Arons 1983; Muslimov \& Harding 2003; Cheng et al. 1986; Romani 1996; Hirotani 2006). Like is the case for radio pulsars, this geometry appears to be very stable. The RXTE monitoring observations proved that the pulse profiles and phase resolved spectra were stable over 9 years. This might indicate that the nonthermal emission is produced higher-up in the magnetosphere, above the region closer to the neutron star with a strong twisted (toroidal) field.

The detailed results we obtained for AXPs 4U 0142+61 and 1RXS J1708-40 clearly prove that the total persistent highenergy emission from AXPs consists of DC emission and pulsed emission exhibiting different spectra (already shown in Kuiper et al. 2006). Furthermore, the pulsed emission consists of multiple components with different spectra. As a consequence it does not seem meaningful to make detailed model fits to just the total AXP spectra to derive a physical interpretation of the emission. The physical origin and production site of each different component has to be considered. Examples of model fitting to the total X-ray spectra of magnetars are recent applications of the resonant cyclotron scattering model first proposed by Thompson et al. (2002). Recent developments of this model can be found in Lyutikov \& Gavriil (2006), Güver et al. (2006, 2007) and Fernández \& Thompson (2007). This model computes the result of multiple scatterings of soft photons in the magnetospheres and was developed to explain the apparently thermal black-body spectra with a power-law extension to higher energies, as measured for energies below $\sim 10 \mathrm{keV}$. In the extensive work by Rea et al. (2008) detailed fits with this model are merely made to the high-energy total spectra of a comprehensive set of magnetars, including 1RXS J170840 , leading to interesting estimates of e.g. optical depths and magnetospheric electron densities at the resonances. However, our temporal and spectral decoupling of different components in the pulsed emission (Figures 10 \& 11), shows that between $\sim 4 \mathrm{keV}$ and $20 \mathrm{keV}$ a peculiar curved spectral component contributes significantly to the pulsed emission, and is unrelated to the soft thermal emission. This component contributes also significantly to the total emission detected from 1RXS J170840 in the energy interval where the resonant upscattering is effective. Hence, it will obviously have an adverse impact on the parameter estimations if this component has not been subtracted. On the other hand, the resonant cyclotron-scattering model might offer a good explanation for the soft pulse peaking at phase 0.4 . Spectral modeling of the corresponding spectrum, and separately of the thermal spectrum of the DC emission will probe the model parameters in different sites in the magnetosphere.

An important parameter to be reproduced by the models is the photon index of the power-law spectral shape in the hard $\mathrm{X}$-ray window and the cut-off energy. The index $\Gamma \sim 1.0$ of the non-thermal pulse and a spectral break at a few hundred $\mathrm{keV}$ is consistent with a bremsstrahlung origin as first proposed in the corona model by Beloborodov \& Thompson (2007). But, further elaboration of the corona model by Lyubarsky \& Eichler (2008) lead to a different hard X-ray spectrum, namely a superposition of bremsstrahlung radiation from the hot atmosphere and Comptonization radiation from the extended corona. The latter produces a hard spectrum up to the $\mathrm{MeV}$ band and is inconsistent with the COMPTEL upper limits at MeV energies. Furthermore, the stability of the configuration over 9 years is a challenge for the corona model. For the resonant upscattering model of Baring \& Harding (2007) the predicted photon indexes are considerably softer and the spectra extend into the $\mathrm{MeV}$ range, possibly to significantly higher gamma-ray 
energies. In this respect we should emphasize that we donot see any indication for a break in the INTEGRAL spectrum $(<300 \mathrm{keV})$ of 1RXS J1708-40, more importantly, we donot know for 1RXS J1708-40 and 4U 0142+61 what the shapes of the spectra are above a few hundred keV. Extreme cases are allowed by the data, e.g. a hard spectrum with index $\Gamma \sim 1.0$ with a sharp cut-off, as well as with a break or bend with a flat spectrum extending underneath the COMPTEL upper limits into the $\mathrm{MeV}$ or even $\mathrm{GeV}$ regions.

Similarly hard spectra with $\Gamma \sim 1.1$ have been measured in the hard X-ray window up to $60-200 \mathrm{keV}$ for several young rotation-powered pulsars, e.g. AX J1838.0-0655, PSR J18460258, PSR J1811-1925, PSR J1930+1852 and PSR J16175055 (Kuiper et al. 2008; Kuiper \& Hermsen 2008). We also know that these hard power-law spectra donot extrapolate to the $\mathrm{MeV}$ range (no detections by COMPTEL). Furthermore, those young pulsars detected with COMPTEL in the MeV range, $\mathrm{Crab}$ (for a multi-wavelength analysis similar to this work see Kuiper et al. 2001) and PSR B1509-58 (Kuiper et al. 1999), have flat $(\Gamma \sim 2)$ spectra at $\mathrm{MeV}$ energies, and started with harder spectra at hard $\mathrm{X}$-ray energies. In addition, for three millisecond pulsars also an index $\Gamma \sim 1.1$ has been measured in the X-ray band up to $25 \mathrm{keV}$ (PSR J0218+4232, PSR B182124 and PSR B1937+21; Kuiper et al. 2004b; Cusumano et al. 2003). For one of these millisecond pulsars, PSR J0218+4232, detection has been claimed even between $300 \mathrm{MeV}$ and $1 \mathrm{GeV}$ with a soft spectral index of $\sim 2.6$ (Kuiper et al. 2000), thus a drastic spectral bend or break is required between the hard Xrays and the high-energy gamma rays. These results on young as well as on old millisecond radio pulsars prove that in dipole fields such hard non-thermal spectra can be produced in stable configurations. The major difference with the AXPs is that the energy source for ordinary pulsars is rotational energy and for AXPs/magnetars magnetic energy. It is interesting to note that recently during a $\sim 50$-day outburst magnetar-like bursts have been reported from a very young rotation-powered pulsar (PSR J1846-0258) with a strong magnetic field (Kumar \& Safi-Harb 2008; Gavriil et al. 2008).

A possible scenario for the persistent high-energy emission from AXPs seems to emerge in which a soft thermal component, for 1 RXS J1708-40 peaking at phase 0.4 , originates in regions above and close to the polar cap of the neutron star with resonant cyclotron scattering offering a physical explanation. A large fraction of the thermal photons will have a more isotropic distribution. These photons can be responsible for the soft part of the DC emission spectrum, also with a resonant cyclotron-scattering origin, but with e.g. different optical depths and magnetospheric electron densities at the resonances. For future studies, it will be interesting to accurately derive a spectrum of just the DC emission for model fitting. Furthermore, the non-thermal emission might be produced higher up in the outer magnetosphere, where the magnetic field strengths is in the range estimated for normal radio pulsars. The required strong current of electrons and positrons can be sustained by the outward transfer of magnetic helicity from the inner zone where it is injected. Then the acceleration of particles and the production of the observed non-thermal photons has to occur in stable narrow zones along the curved field lines to produce e.g. the narrow hard X-ray pulse seen for 1RXS J1708-40. In the outer magnetosphere the scenario might resemble those proposed for the high-energy emission from rotation-powered pulsars. In fact, the approach taken by Baring \& Harding (2007) is a first attempt along these lines. However, they concluded that the resonant magnetic Compton upscattering is most effective close to the magnetar surface.

From only two of the 13 confirmed magnetars radio emission has been detected and their radio emission properties have been measured in great detail recently, notably from AXP XTE J1810-197 Halpern et al. (2005); Camilo et al. (2006); Kramer et al. (2007) and AXP 1E 1547.0-5408 (Camilo et al. 2007, 2008). These very interesting AXPs show transient radio emission, which is apparently related to the X-ray variability (e.g. Ibrahim et al. 2004; Gotthelf \& Halpern 2005; Gelfand \& Gaensler 2007; Halpern et al. 2008). These radio AXPs appear to be very highly linearly polarized and have very flat spectra over a wide range of radio frequencies, very similar characteristics to those of many young ordinary radio pulsars. Camilo et al. (2008) note that, while unexpected a priori, it appears that ideas developed to understand the geometry of emission from ordinary pulsars along open dipolar field lines also apply to known radio magnetars. However, they also conclude that both radio AXPs have also variable pulse profiles and radio flux densities, not typical for young pulsars.

Upon submission of this work, an extensive treatment appeared of pair creation processes in an ultra-strong magnetic field and particle heating in a dynamic magnetosphere (Thompson 2008a), as well as a self-consistent model of the inner accelerator with implications for pulsed radio emission (Thompson 2008b). In the first paper Thompson considers the details of QED processes that create electron-positron pairs in magnetar fields. He discusses how the pair creation rate in the open-field circuit and the outer magnetosphere can be strongly enhanced by instabilities near the light cylinder and how this very high rate of pair creation on the open magnetic field lines can help to stabilize a static twist in the closed magnetosphere and to regulate the loss of magnetic helicity by reconnection at the light cylinder. In the second paper, one of the possible scenarios for explaining the rapid radio variability, broad pulses and hard radio spectra comprises a strongly variable current in the outer magnetosphere and a high rate of pair creation sustained by a turbulent cascade. It is interesting to note that stable geometries are being considered to explain the production of transient radio emission for AXPs involving high rates of pair creation on the open magnetic-field lines in the outer magnetosphere. Such scenarios might also be required to explain the production in the outer magnetosphere on the orders of magnitude more luminous non-thermal X-ray emission.

Acknowledgements. We thank Jörgen Knödlseder for providing the SPI upper limits for 1RXS J1708-40. This work is supported by NWO, Netherlands Organisation for Scientific Research. The results are based on observations with INTEGRAL, an ESA project with instruments and science data centre funded by ESA member states (especially the PI countries: Denmark, France, Germany, Italy, Switzerland, Spain), Czech Republic and Poland, and with the participation of Russia and the USA. We are grateful to ASI, CEA, CNES, DLR, ESA, INTA, NASA and OSTC for support. This research has made use of 
data obtained through the High-Energy Astrophysics Center Online Service, provided by the NASA/Goddard Space-Flight Center.

\section{References}

Arnaud, K. A. 1996, in ASP Conf. Ser. 101: Astronomical Data Analysis Software and Systems V, 17-20

Arons, J. 1983, ApJ, 266, 215

Baring, M. G. \& Harding, A. K. 2007, Ap\&SS, 308, 109

Beloborodov, A. M. \& Thompson, C. 2007, ApJ, 657, 967

Buccheri, R., Bennett, K., Bignami, G. F., et al. 1983, A\&A, 128,245

Camilo, F., Ransom, S. M., Halpern, J. P., \& Reynolds, J. 2007, ApJ, 666, L93

Camilo, F., Ransom, S. M., Halpern, J. P., et al. 2006, Nature, 442, 892

Camilo, F., Reynolds, J., Johnston, S., Halpern, J. P., \& Ransom, S. M. 2008, accepted for ApJ, astro-ph/0802.0494

Campana, S., Rea, N., Israel, G. L., Turolla, R., \& Zane, S. 2007, A\&A, 463, 1047

Cheng, K. S., Ho, C., \& Ruderman, M. 1986, ApJ, 300, 500

Cusumano, G., Hermsen, W., Kramer, M., et al. 2003, A\&A, 410, L9

den Hartog, P. R., Hermsen, W., Kuiper, L., et al. 2008, accepted for A\&A

den Hartog, P. R., Hermsen, W., Kuiper, L., et al. 2006, A\&A, 451,587

den Hartog, P. R., Kuiper, L., Hermsen, W., \& Vink, J. 2004, The Astronomer's Telegram, 293, 1

den Herder, J. W., Brinkman, A. C., Kahn, S. M., et al. 2001, A\&A, 365, L7

Dib, R., Kaspi, V. M., \& Gavriil, F. P. 2008, ApJ, 673, 1044

Duncan, R. C. \& Thompson, C. 1992, ApJ, 392, L9

Durant, M. \& van Kerkwijk, M. H. 2006a, ApJ, 650, 1070

Durant, M. \& van Kerkwijk, M. H. 2006b, ApJ, 650, 1082

Eadie, W. T., Drijard, D., \& James, F. E. 1971, Statistical methods in experimental physics (Amsterdam: North-Holland, 1971)

Fernández, R. \& Thompson, C. 2007, ApJ, 660, 615

Gavriil, F. P., Gonzalez, M. E., Gotthelf, E. V., et al. 2008, accepted for Science; astro-ph/0802.1704

Gavriil, F. P. \& Kaspi, V. M. 2002, ApJ, 567, 1067

Gelfand, J. D. \& Gaensler, B. M. 2007, ApJ, 667, 1111

Goldwurm, A., David, P., Foschini, L., et al. 2003, A\&A, 411, L223

Gotthelf, E. V. \& Halpern, J. P. 2005, ApJ, 632, 1075

Götz, D., Rea, N., Israel, G. L., et al. 2007, A\&A, 475, 317

Güver, T., Özel, F., Göğüş, E., \& Kouveliotou, C. 2007, ApJ, 667, L73

Güver, T., Özel, F., \& Lyutikov, M. 2006, submitted to ApJ; astro-ph/0611405

Halpern, J. P., Gotthelf, E. V., Becker, R. H., Helfand, D. J., \& White, R. L. 2005, ApJ, 632, L29

Halpern, J. P., Gotthelf, E. V., Reynolds, J., Ransom, S. M., \& Camilo, F. 2008, accepted for ApJ; astro-ph/0711.3780

Harding, A. K. \& Muslimov, A. G. 1998, ApJ, 508, 328

Heyl, J. S. 2007, Ap\&SS, 308, 101

Heyl, J. S. \& Hernquist, L. 2005a, ApJ, 618, 463
Heyl, J. S. \& Hernquist, L. 2005b, MNRAS, 362, 777

Hirotani, K. 2006, ApJ, 652, 1475

Ibrahim, A. I., Markwardt, C. B., Swank, J. H., et al. 2004, ApJ, 609, L21

Israel, G., Oosterbroek, T., Stella, L., et al. 2001, ApJ, 560, L65

Israel, G. L., Covino, S., Perna, R., et al. 2003, ApJ, 589, L93

Israel, G. L., Götz, D., Zane, S., et al. 2007, A\&A, 476, L9

Jahoda, K., Swank, J. H., Giles, A. B., et al. 1996, in Proc. SPIE Vol. 2808, p. 59-70, EUV, X-Ray, and Gamma-Ray Instrumentation for Astronomy VII, Oswald H. Siegmund; Mark A. Gummin; Eds., ed. O. H. Siegmund \& M. A. Gummin, 59-70

Jansen, F., Lumb, D., Altieri, B., et al. 2001, A\&A, 365, L1

Jensen, P. L., Clausen, K., Cassi, C., et al. 2003, A\&A, 411, L7

Kaspi, V. M. 2007, Ap\&SS, 308, 1

Kaspi, V. M., Chakrabarty, D., \& Steinberger, J. 1999, ApJ, 525, L33

Knödlseder, J. 2004, in ESA Special Publication, Vol. 552, 5 th INTEGRAL Workshop on the INTEGRAL Universe, ed. V. Schoenfelder, G. Lichti, \& C. Winkler, 867

Kramer, M., Stappers, B. W., Jessner, A., Lyne, A. G., \& Jordan, C. A. 2007, MNRAS, 377, 107

Kuiper, L. \& Hermsen, W. 2008, in prep. for A\&A

Kuiper, L., Hermsen, W., Cusumano, G., et al. 2001, A\&A, 378,918

Kuiper, L., Hermsen, W., den Hartog, P. R., \& Collmar, W. 2006, ApJ, 645, 556

Kuiper, L., Hermsen, W., Klein-Wolt, M., \& Wijnands, R. 2008, The Astronomer's Telegram, 1405, 1

Kuiper, L., Hermsen, W., Krijger, J. M., et al. 1999, A\&A, 351, 119

Kuiper, L., Hermsen, W., \& Mendez, M. 2004a, ApJ, 613, 1173

Kuiper, L., Hermsen, W., \& Stappers, B. 2004b, Advances in Space Research, 33, 507

Kuiper, L., Hermsen, W., Verbunt, F., et al. 2000, A\&A, 359, 615

Kumar, H. S. \& Safi-Harb, S. 2008, accepted for ApJ; astro$\mathrm{ph} / 0802.1242$

Lebrun, F., Leray, J. P., Lavocat, P., et al. 2003, A\&A, 411, L141

Lyubarsky, Y. \& Eichler, D. 2008, submitted to ApJ; astro$\mathrm{ph} / 0706.3578$

Lyutikov, M. \& Gavriil, F. P. 2006, MNRAS, 368, 690

Mereghetti, S., Esposito, P., \& Tiengo, A. 2007, Ap\&SS, 308, 13

Molkov, S. V., Cherepashchuk, A. M., Lutovinov, A. A., et al. 2004, Astronomy Letters, 30, 534

Muslimov, A. G. \& Harding, A. K. 2003, ApJ, 588, 430

Rea, N., Israel, G. L., Oosterbroek, T., et al. 2007, Ap\&SS, 308, 505

Rea, N., Oosterbroek, T., Zane, S., et al. 2005, MNRAS, 361, 710

Rea, N., Zane, S., Turolla, R., Lyutikov, M., \& Gotz, D. 2008, submitted to ApJ, astro-ph/0802.1923

Revnivtsev, M. G., Sunyaev, R. A., Varshalovich, D. A., et al. 2004, Astronomy Letters, 30, 382

Romani, R. W. 1996, ApJ, 470, 469

Rothschild, R. E., Blanco, P. R., Gruber, D. E., et al. 1998, ApJ, 
496, 538

Ruderman, M. A. \& Sutherland, P. G. 1975, ApJ, 196, 51

Strüder, L., Briel, U., Dennerl, K., et al. 2001, A\&A, 365, L18

Sugizaki, M., Nagase, F., Torii, K., et al. 1997, PASJ, 49, L25

Thompson, C. 2008a, submitted to ApJ, astro-ph/0802.2571

Thompson, C. 2008b, submitted to ApJ, astro-ph/0802.2572

Thompson, C. \& Duncan, R. C. 1993, ApJ, 408, 194

Thompson, C. \& Duncan, R. C. 1995, MNRAS, 275, 255

Thompson, C. \& Duncan, R. C. 1996, ApJ, 473, 322

Thompson, C., Lyutikov, M., \& Kulkarni, S. R. 2002, ApJ, 574, 332

Turner, M. J. L., Abbey, A., Arnaud, M., et al. 2001, A\&A, $365, \mathrm{~L} 27$

Ubertini, P., Lebrun, F., Di Cocco, G., et al. 2003, A\&A, 411, L131

Usov, V. V. \& Melrose, D. B. 1995, Australian Journal of Physics, 48, 571

Valinia, A. \& Marshall, F. E. 1998, ApJ, 505, 134

Vedrenne, G., Roques, J.-P., Schönfelder, V., et al. 2003, A\&A, 411, L63

Walter, R., Favre, P., Dubath, P., et al. 2003, A\&A, 411, L25

Woods, P. M. \& Thompson, C. 2006, in Cambridge Astrophysics Series, Vol. 39, Compact stellar X-ray sources, ed. W. Lewin \& M. van der Klis, 547 